\title{
Determination of 40 Elements in Powdered Infant Formulas and Related Risk Assessment
}

\author{
Maria Luisa Astolfi ${ }^{1}$ (D, Daniela Marotta ${ }^{2}$, Vittoria Cammalleri ${ }^{2}$, Elisabetta Marconi ${ }^{3}$, Arianna Antonucci ${ }^{2}$, \\ Pasquale Avino ${ }^{4}$ (D) Silvia Canepari ${ }^{5}$ (D), Matteo Vitali ${ }^{2}$ (D) and Carmela Protano ${ }^{2, *(D)}$
}

\section{check for}

updates

Citation: Astolfi, M.L.; Marotta, D. Cammalleri, V.; Marconi, E.;

Antonucci, A.; Avino, P.; Canepari, S.;

Vitali, M.; Protano, C. Determination

of 40 Elements in Powdered Infant

Formulas and Related Risk

Assessment. Int. J. Environ. Res.

Public Health 2021, 18, 5073.

https://doi.org/10.3390/

ijerph18105073

Academic Editor: Paul B. Tchounwou

Received: 15 April 2021

Accepted: 9 May 2021

Published: 11 May 2021

Publisher's Note: MDPI stays neutral with regard to jurisdictional claims in published maps and institutional affiliations.

Copyright: (c) 2021 by the authors. Licensee MDPI, Basel, Switzerland. This article is an open access article distributed under the terms and conditions of the Creative Commons Attribution (CC BY) license (https:// creativecommons.org/licenses/by/ $4.0 /)$
1 Department of Chemistry, "Sapienza" University of Rome, 00185 Rome, Italy; marialuisa.astolfi@uniroma1.it

2 Department of Public Health and Infectious Diseases, "Sapienza” University of Rome, 00185 Rome, Italy; daniela.marotta@uniroma1.it (D.M.); vittoria.cammalleri@uniroma1.it (V.C.); arianna.antonucci@uniroma1.it (A.A.); matteo.vitali@uniroma1.it (M.V.)

3 National Research Council (CNR), Institute of Atmospheric Pollution Research, Via Salaria Km. 29.300, Monterotondo St., 00015 Rome, Italy; elisabetta.marconi@iia.cnr.it

4 Department of Agricultural, Environmental and Food Sciences (DiAAA), University of Molise, Via F. De Sanctis, 86100 Campobasso, Italy; avino@unimol.it

5 Department of Environmental Biology, "Sapienza" University of Rome, 00185 Rome, Italy; silvia.canepari@uniroma1.it

* Correspondence: carmela.protano@uniroma1.it

Abstract: The aim of the study was to analyze all powdered infant formulas authorized and commercialized in Italy at the time of the study to measure the concentrations of 40 elements, and to estimate the infants' intake of some toxic heavy metals for assessing possible related health risks. For this purpose, an optimized multi-element method was used through inductively coupled plasma mass spectrometry. Be, B, Al, $\mathrm{Zr}, \mathrm{Nb}, \mathrm{Sb}, \mathrm{Te}, \mathrm{W}, \mathrm{V}, \mathrm{Cr}$ and As concentrations were <LD in more than $30 \%$ of samples. The levels of the other elements resulted to be very variable (more than $2000 \mu \mathrm{g} \mathrm{g}^{-1}$ for $\mathrm{Ca}$ and $\mathrm{K}$ or less than $1 \mathrm{ng} \mathrm{g}^{-1}$ for others). The results were similar to those reported by other European Union (EU) studies, but different from those recovered outside the EU. These differences should be eliminated to guarantee the right to health worldwide. The concentrations of $\mathrm{Cd}, \mathrm{Mn}, \mathrm{Ni}, \mathrm{Pb}$, and $\mathrm{Zn}$ in the infant formulas studied were always below the considered limits. However, it is important to check for potentially toxic elements in infant formulas to protect the health of this sensitive population. The data found in this study could be used as benchmark data for future research.

Keywords: powdered infant formula; inorganic elements; inductively coupled plasma mass spectrometry; daily intake; health risk assessment

\section{Introduction}

Food safety is an issue of great interest for public health, as demonstrated by the robust regulation, the World Health Organization (WHO) recommendations and many scientific research types in the field [1-3]. However, due to the complexity of the topic, food safety is still the subject of toxicological and health threat evaluations, both for microbiological and chemical risks. Particular and specific attention is given to infant feeding [4]. Scientific institutions and associations that deal with children's health emphasize the importance of breastfeeding as long as possible [5-7]. WHO strongly recommends exclusive breastfeeding for the first six months of life to ensure optimal growth, development, and health [8]. Breast milk provides all the nutrients, vitamins and minerals. At the same time, breastfeeding satisfies the emotional and psychological needs of the newborn and creates a special bond between mother and child with positive repercussions for life [9]. When breastfeeding is not possible, infant formulas are the essential alternative to support the newborn growth [8]. They are defined as food products intended for feeding infants in the first months of life and are prepared to satisfy, by itself, the nutritional needs of infants until the introduction 
of adequate complementary nutrition [10]. These formulas are the only products that can be used as substitutes. Thus, the microbiological and chemical safety of infant formulas is essential to protect the newborn's health, and it requires careful evaluation and specific criteria to ensure the highest quality [11]. As regards to the chemical quality, limits set for contaminants or nutrient levels recommended for the adult population cannot be extended to infants since diet, energy requirements and consumption of nutrients are entirely different. Therefore, risk assessments made for infants' diet should be specifically dedicated. The Standard for Infant Formula and Formulas for Special Medical Purposes Intended for Infants CXS 72-1981 [12] contains provisions for essential composition, quality, and safety factors and constitutes the international reference for these products. However, in some countries, regulations have been developed by leading authorities such as the European Commission [10]; as a result, estimated product specifications may differ from country to country [13]. Specific regulations impose minimum and maximum levels for some essential elements ( $\mathrm{Na}, \mathrm{K}, \mathrm{Cl}, \mathrm{Ca}, \mathrm{P}, \mathrm{Mg}, \mathrm{Fe}, \mathrm{Zn}, \mathrm{Cu}, \mathrm{I}, \mathrm{Se}, \mathrm{Mn}$, and $\mathrm{F}$ ) and maximum limits for some inorganic contaminants ( $\mathrm{As}, \mathrm{Cd}, \mathrm{Pb}, \mathrm{Hg}$, and $\mathrm{Sn}$ ) [14]. $\mathrm{Cd}, \mathrm{Cr}, \mathrm{Mn}, \mathrm{Ni}, \mathrm{Pb}$, and $\mathrm{Zn}$ are heavy metals of great concern because they can bio-accumulate in vital organs, persist for long periods of time in the human body, and can determine several negative outcomes for human health, already in the early stages of life [15-17]. The intake of Cd is closely related to adverse effects on the kidney, central nervous system, and alterations in Ca metabolism [18]. The excess Mn intake can cause significant neurotoxic effects for early brain development $[19,20]$. Ni, a known genotoxic and carcinogen, is toxic for hematological, immunological, neurological, pulmonary, reproductive systems [21]. $\mathrm{Pb}$, even at low concentrations, affects infant cognitive and neurobehavioral development [15]. Cr and $\mathrm{Zn}$ are essential micronutrients but, when taken in excess, can determine negative consequences for human health [22]. Besides, currently, legislation dedicated to infant formulas prescribes limits for a few number of elements and does not take into consideration some other ones, such as $\mathrm{Al}$, which was found in high concentrations in some types of formulas [23]. In these cases, it is necessary to respect the precautionary principle to ensure the maximum protection of a particularly susceptible population such as infants [24].

Due to the wide range of chemicals to be determined and the need for dedicated risk assessment, a research agenda useful both for the scientific community and food companies is the development of multi-analyte methods to provide reliable and rapid results at low costs.

The aim of the present study was to determine 40 elements in all the powdered infant formulas authorized and commercialized in Italy at the time of the study by inductively coupled plasma mass spectrometer (ICP-MS). Besides, infants' intake of some elements of relevance for human health $(\mathrm{Cd}, \mathrm{Cr}, \mathrm{Mn}, \mathrm{Ni}, \mathrm{Pb}$ and $\mathrm{Zn})$ was estimated to assess the possible related health risks.

\section{Materials and Methods}

\subsection{Sample Collection and Preparation}

The selected powdered infant formulas were included in the National Register of products defined by the Italian Ministry of Health and established according to the Ministerial Decree of 17 May 2016 [25]. Indeed, all infant formulas must be entered in the National Register to be marketed in Italy. At the time of the study's conduction (April 2019), the National Register included 16 powdered and three liquid formulas. A total of 11 powdered infant formulas were collected from the local pharmacies, while five products were not available. We purchased two packs of powdered milk of each marketed product for a total of 22 packs.

Each sample of the studied formula was accurately weighed ( $\sim .5 \mathrm{~g})$ using an analytical balance (Europe 60; Gibertini Elettronica, Milan, Italy) and single-use weighing boats. Each sample was placed in a polyethylene tube and carried out a "weighted by difference"; that is, the first weight of the sample subtracted from the weight of what is left in the weighing boats. This process was carried out twice, and the tubes were labeled as A 
and B. Each tube was analyzed in triplicate and all the results from the two packs were used to calculate the final mean of each studied formula.

In a second phase, $0.25 \mathrm{~mL}$ of $30 \% \mathrm{H}_{2} \mathrm{O}_{2}$ super-pure and $0.5 \mathrm{~mL}$ of $67 \% \mathrm{HNO}_{3}$ superpure (Promochem, LGC Standards GmbH, Wesel, Germany) were added to each test tube using micropipettes (Gilson, Middleton, WI, USA), changing the disposable tip each time. The samples were digested using a water bath (WB12; Argo Lab, Modena, Italy) at $95{ }^{\circ} \mathrm{C}$ for $20 \mathrm{~min}$ [26]. Then, the test tubes were left to cool, and the content was diluted to $10 \mathrm{~mL}$ with deionized water. Samples were then filtered using a $0.45 \mu \mathrm{m}$ microcellulose filter (GVS Life Sciences, GVS North America, Sanford, ME, USA) pre-washed with 1\% ( $v / v)$ $\mathrm{HNO}_{3}$. About $2 \mathrm{~mL}$ were discarded for each sample, while the remaining volume was put in another test tube and then inserted into the test holder for analytical determinations.

The method accuracy was checked through spike samples standard reference material (SRM 1954; National Institute of Standards and Technology, Gaithersburg, MD, USA), and European reference materials $\left(\mathrm{ERM}^{\circledR}{ }^{\circledR}-\mathrm{BD} 150\right.$ and ERM ${ }^{\circledR}$-BD151, Joint Research Centre, Institute for Reference Materials and Measurements, Geel, Belgium), used as previously reported [27-29]. Briefly, the certified material was reconstituted according to the manufacturer's recommendations and analyzed after calibration. There was a good agreement between the obtained results and certified values, with trueness bias percentages ranging from -7 to $8 \%$ and precision $<5 \%$. Acceptable recoveries ranged from 91 to $107 \%$ and precision $<15 \%$ were obtained by spiked cow milk samples. Method blanks were prepared by diluting a proper amount of $1 \%(v / v) \mathrm{HNO}_{3}$ in polyethylene tubes.

\subsection{Instrumentation}

An ICP-MS (820-MS Bruker, Bremen, Germany), equipped with a glass nebulizer (0.4 mL min ${ }^{-1}$; MicroMistTM) and a collision reaction interface (CRI) with $\mathrm{He}$ and $\mathrm{H}_{2}$ (99.9995\% purity; SOL Spa, Monza, Italy) was used for multi-element analysis.

A solution of $Y\left(5 \mu \mathrm{g} \mathrm{L}^{-1}\right.$ from $1000 \pm 2 \mathrm{mg} \mathrm{L}^{-1}$; Panreac Quimica, Barcelona, Spain), Sc, Rh, Th and In ( $10 \mu \mathrm{g} \mathrm{L}{ }^{-1}$ from $1000 \pm 2 \mathrm{mg} \mathrm{L}^{-1}$, Panreac Quimica, Barcelona, Spain) in $2 \%(v / v) \mathrm{HNO}_{3}[29]$ was used as internal standards.

\subsection{Quality Assurance}

The limits of detection (LODs) and quantification (LOQs) were calculated, respectively, as three and ten times the blank sample's standard deviation (six replicates). For each element, arithmetic means (AMs) and standard deviations (SDs) were calculated (three replicates); when the result was lower than the LOD, it was considered LOD/2. When the single element results were below the LOD for more than $30 \%$ of samples, the element was excluded from subsequent elaboration.

\subsection{Risk Evaluation}

The daily intake for each studied heavy metal $(\mathrm{Cd}, \mathrm{Cr}, \mathrm{Mn}, \mathrm{Ni}, \mathrm{Pb}$, and $\mathrm{Zn})$ was estimated considering the concentration of the metal obtained from the analysis of the samples, the average daily/weekly intake of the formula, and the average body weight (bw) for males and females separately, and considering different time intervals from birth to 6 months old according to the nutrition requirements specific for each period of life.

The metal concentration was directly derived from the analysis as the means of the values obtained from all the analyzed formulas, as previously performed by Eticha et al., 2018 [30]. Daily doses were calculated using the infant's feed tables. The average bw was determined according to the child growth standards charts developed by WHO [31], considering the 50th percentile of the weight for males and females at 1st week (for the period of life of 0-2 weeks), 3rd week (for 2-4 weeks), 1st month (for 2 months), 4th month (for 4 months) and 6th month (for 6 months). The daily/weekly intake for each metal was calculated by the following equation:

$$
\text { Daily intake }\left(\mu \mathrm{g} \mathrm{kg}^{-1} \mathrm{bw}\right)=\frac{\mathrm{Cm} \times \mathrm{EI}}{\mathrm{bw}}
$$


$\mathrm{Cm}$ is the mean concentration of each studied metal in the formulas, expressed as $\mu \mathrm{g} \mathrm{g}^{-1}$;

EI is the daily or weekly estimated intake of formulas expressed as g;

bw is the body weight expressed as $\mathrm{kg}$.

Finally, each considered metal's health risk index was calculated as a percentage of its safety limit. The safety limits were as follows: for Cd, the European Food Safety Authority (EFSA) Panel on Contaminants in the Food Chain indicates a provisional tolerable weekly intake (PTWI) of $2.5 \mu \mathrm{g} \mathrm{kg}{ }^{-1} \mathrm{bw} /$ week [32,33]; for Pb, the Joint FAO/WHO Expert Committee on Food Additives (JECFA) reports a PTWI equal to $3.5 \mu \mathrm{g} \mathrm{kg}^{-1} \mathrm{bw} /$ week [34]; for Zn, the Scientific Committee on Food (SCF) indicates a tolerable upper limit of $7 \mathrm{mg} /$ day [35]. A total of 300 and $2.8 \mu \mathrm{g} \mathrm{kg}^{-1} \mathrm{bw} /$ day were recommended by the EFSA as the provisional tolerable daily intake (PTDI) for $\mathrm{Cr}$ and $\mathrm{Ni}$, respectively [36,37].

As regards to $\mathrm{Mn}$, we considered the current $\mathrm{EU}$ and French regulatory minima and maxima values for infant and follow-on formulas, that stipulated a minimum content of $1 \mu \mathrm{g}$ of Mn/100 kcal and a maximum content of $100 \mu \mathrm{g}$ of Mn/100 kcal [38,39]. In order to compare our results with regulatory values, we converted the minimum and maximum level from $\mu \mathrm{g} \mathrm{kcal}^{-1}$ to $\mu \mathrm{g} \mathrm{g}^{-1}$ by calculating the mean $\mathrm{kcal}$ content of $1 \mathrm{~g}$ of all the studied formulas, that resulted equal to $5.04 \mathrm{kcal}$ per gram of formula; thus, the regulatory limits resulted 0.0504 and $5.04 \mu \mathrm{g} \mathrm{Mn} \mathrm{g}^{-1}$ formula, respectively.

\section{Results}

Table 1 shows the limits of determination (LODs) and the percentages below the LOD for the 40 elements analyzed in this study.

Table 1. Selection of isotopes, limit of determination (LOD) expressed as $\mu \mathrm{g} \mathrm{g}^{-1}$ formula and percentage of values lower than LOD for each element.

\begin{tabular}{ccc}
\hline Element & LOD & \% < LOD \\
\hline${ }^{7} \mathrm{Li}$ & 0.00005 & 0 \\
${ }^{9} \mathrm{Be}$ & 0.0001 & 82 \\
${ }^{11} \mathrm{~B}$ & 0.6 & 100 \\
${ }^{23} \mathrm{Na}$ & 4 & 0 \\
${ }^{24} \mathrm{Mg}$ & 0.3 & 0 \\
${ }^{27} \mathrm{Al}$ & 0.01 & 73 \\
${ }^{28} \mathrm{Si}$ & 1 & 0 \\
${ }^{31} \mathrm{P}$ & 4 & 0 \\
${ }^{39} \mathrm{~K}$ & 5 & 0 \\
${ }^{44} \mathrm{Ca}$ & 3 & 0 \\
${ }^{49} \mathrm{Ti}$ & 0.002 & 0 \\
${ }^{59} \mathrm{Co}$ & 0.0003 & 0 \\
${ }^{60} \mathrm{Ni}$ & 0.02 & 0 \\
${ }^{65} \mathrm{Cu}$ & 0.01 & 0 \\
${ }^{66} \mathrm{Zn}$ & 0.1 & 0 \\
${ }^{71} \mathrm{Ga}$ & 0.0004 & 0 \\
${ }^{85} \mathrm{Rb}$ & 0.001 & 0 \\
${ }^{88} \mathrm{Sr}$ & 0.01 & 0 \\
${ }^{90} \mathrm{Zr}$ & 0.002 & 59 \\
${ }^{93} \mathrm{Nb}$ & 0.01 & 100 \\
${ }^{98} \mathrm{Mo}$ & 0.004 & 0 \\
${ }^{112} \mathrm{Cd}$ & 0.0004 & 97 \\
${ }^{118} \mathrm{Sn}$ & 0.0002 & 0 \\
${ }^{121} \mathrm{Sb}$ & 0.01 & 77 \\
\hline
\end{tabular}


Table 1. Cont.

\begin{tabular}{ccc}
\hline Element & LOD & $\%<$ LOD \\
\hline${ }^{125} \mathrm{Te}$ & 0.003 & 91 \\
${ }^{133} \mathrm{Cs}$ & 0.00005 & 0 \\
${ }^{137} \mathrm{Ba}$ & 0.01 & 0 \\
${ }^{139} \mathrm{La}$ & 0.00003 & 0 \\
${ }^{140} \mathrm{Ce}$ & 0.0001 & 0 \\
${ }^{182} \mathrm{~W}$ & 0.002 & 50 \\
${ }^{205} \mathrm{Tl}$ & 0.0001 & 5 \\
${ }^{208} \mathrm{~Pb}$ & 0.001 & 18 \\
${ }^{209} \mathrm{Bi}$ & 0.001 & 23 \\
${ }^{238} \mathrm{U}$ & 0.00003 & 0 \\
${ }^{51} \mathrm{~V}$ & 0.002 & 36 \\
${ }^{52} \mathrm{Cr}$ & 0.01 & 32 \\
${ }^{55} \mathrm{Mn}$ & 0.004 & 0 \\
${ }^{56} \mathrm{Fe}$ & 0.1 & 0 \\
${ }^{75} \mathrm{As}$ & 0.01 & 77 \\
${ }^{76} \mathrm{Se}$ & 0.01 & 0 \\
\hline
\end{tabular}

$\mathrm{Be}, \mathrm{B}, \mathrm{Al}, \mathrm{Zr}, \mathrm{Nb}, \mathrm{Sb}, \mathrm{Te}, \mathrm{W}, \mathrm{V}, \mathrm{Cr}$, and As were excluded from the following analysis because, in the 11 infant formulas considered in the present study, these elements were detected in concentrations lower than the LOD in more than $30 \%$ of cases.

The descriptive statistics (AM and the $\mathrm{SD}$, median and interquartile range- $\mathrm{IQR}$ ) of the remaining element concentrations were calculated for each formula and are reported in Tables 2-6.

The values obtained by the analytical determinations resulted to be extremely variable, ranging between more than $2000 \mathrm{\mu g} \mathrm{g}^{-1}$ for $\mathrm{Ca}$ and $\mathrm{K}$ and less than $1 \mathrm{ng} \mathrm{g}^{-1}$ for elements such as $\mathrm{Tl}$ and $\mathrm{U}$. Besides, we recovered different concentrations for each element in the 11 considered formulas.

Figure 1 shows the mean concentrations, expressed as $\mu \mathrm{g} \mathrm{g}^{-1}$ for each formula, of the elements selected for the risk assessment $(\mathrm{Ni}, \mathrm{Zn}, \mathrm{Cd}, \mathrm{Pb}) . \mathrm{Cr}$ and $\mathrm{Mn}$ are not reported in Figure 1 because more than 30\% of the values of $\mathrm{Cr}$ were below the LOD and the risk evaluation for $\mathrm{Mn}$ was performed comparing its concentrations with the minima and maxima values stipulated by EU and French regulations. 
Table 2. Descriptive statistics of $\mathrm{Li}, \mathrm{Na}, \mathrm{Mg}, \mathrm{Si}, \mathrm{P}$ and $\mathrm{K}$ calculated on a total of 12 determinations for each formula and expressed as $\mu \mathrm{g} \mathrm{g}^{-1}$.

\begin{tabular}{|c|c|c|c|c|c|c|c|c|c|c|c|c|}
\hline \multirow[b]{2}{*}{$\begin{array}{l}\text { Infant } \\
\text { Formula }\end{array}$} & \multicolumn{2}{|c|}{$\mathbf{L i}$} & \multicolumn{2}{|c|}{$\mathrm{Na}$} & \multicolumn{2}{|c|}{$\mathrm{Mg}$} & \multicolumn{2}{|c|}{$\mathrm{Si}$} & \multicolumn{2}{|c|}{$\mathbf{P}$} & \multicolumn{2}{|c|}{$\mathrm{K}$} \\
\hline & $\mathrm{AM} \pm \mathrm{SD}$ & $\begin{array}{c}\text { Median } \\
\text { IQR }\end{array}$ & $\mathbf{A M} \pm \mathbf{S D}$ & $\begin{array}{c}\text { Median } \\
\text { IQR }\end{array}$ & $\mathbf{A M} \pm \mathbf{S D}$ & $\begin{array}{c}\text { Median } \\
\text { IQR }\end{array}$ & $\mathbf{A M} \pm \mathbf{S D}$ & $\begin{array}{c}\text { Median } \\
\text { IQR }\end{array}$ & $\mathbf{A M} \pm \mathbf{S D}$ & $\begin{array}{c}\text { Median } \\
\text { IQR }\end{array}$ & $\mathbf{A M} \pm \mathbf{S D}$ & $\begin{array}{c}\text { Median } \\
\text { IQR }\end{array}$ \\
\hline 1 & $0.0060 \pm 0.001$ & $\begin{array}{c}0.00603 \\
0.00581-0.00626\end{array}$ & $635 \pm 37$ & $\begin{array}{c}634.8 \\
621.6-648.0\end{array}$ & $204 \pm 14$ & $\begin{array}{c}204.2 \\
199.4-209.0\end{array}$ & $22.0 \pm 1.3$ & $\begin{array}{c}22.03 \\
21.57-22.50\end{array}$ & $770 \pm 50$ & $\begin{array}{c}770.2 \\
752.6-787.9\end{array}$ & $2439 \pm 154$ & $\begin{array}{c}2438.6 \\
2384.1-2493.1\end{array}$ \\
\hline 3 & $0.0037 \pm 0.0001$ & $\begin{array}{c}0.00369 \\
0.00364-0.00374\end{array}$ & $687 \pm 25$ & $\begin{array}{c}687.1 \\
678.3-695.9\end{array}$ & $133 \pm 3$ & $\begin{array}{c}133.4 \\
132.3-134.5\end{array}$ & $19.7 \pm 0.9$ & $\begin{array}{c}19.66 \\
19.36-19.97\end{array}$ & $906 \pm 23$ & $\begin{array}{c}906.2 \\
898.0-914.5\end{array}$ & $2085 \pm 53$ & $\begin{array}{c}2085.2 \\
2066.5-2103.9\end{array}$ \\
\hline 4 & $0.0076 \pm 0.0003$ & $\begin{array}{c}0.00758 \\
0.00747-0.00769\end{array}$ & $583 \pm 19$ & $\begin{array}{c}582.7 \\
576.2-589.2\end{array}$ & $161 \pm 4$ & $\begin{array}{c}160.9 \\
159.5-162.3\end{array}$ & $22.5 \pm 0.3$ & $\begin{array}{c}22.54 \\
22.42-22.66\end{array}$ & $699 \pm 13$ & $\begin{array}{c}699.2 \\
694.7-703.7\end{array}$ & $2518 \pm 54$ & $\begin{array}{c}2517.5 \\
2498.5-2536.5\end{array}$ \\
\hline 5 & $0.0098 \pm 0.0008$ & $\begin{array}{c}0.00985 \\
0.00955-0.01014\end{array}$ & $715 \pm 73$ & $\begin{array}{c}715.3 \\
689.4-741.1\end{array}$ & $281 \pm 37$ & $\begin{array}{c}281.0 \\
268.0-294.1\end{array}$ & $18.9 \pm 1.2$ & $\begin{array}{c}18.91 \\
18.50-19.33\end{array}$ & $544 \pm 46$ & $\begin{array}{c}543.5 \\
527.3-559.6\end{array}$ & $2578 \pm 210$ & $\begin{array}{c}2578.1 \\
2505.3-2651.0\end{array}$ \\
\hline 6 & $0.0074 \pm 0.0002$ & $\begin{array}{c}0.00741 \\
0.00736-0.00747\end{array}$ & $619 \pm 20$ & $\begin{array}{c}618.6 \\
611.6-625.5\end{array}$ & $167 \pm 5$ & $\begin{array}{c}167.4 \\
165.7-169.1\end{array}$ & $18.4 \pm 0.3$ & $\begin{array}{c}18.43 \\
18.33-18.52\end{array}$ & $817 \pm 8$ & $\begin{array}{c}817.2 \\
814.2-820.2\end{array}$ & $2169 \pm 35$ & $\begin{array}{c}2169.1 \\
2156.6-2181.5\end{array}$ \\
\hline 7 & $0.0055 \pm 0.0001$ & $\begin{array}{c}0.00547 \\
0.00546-0.00549\end{array}$ & $535 \pm 6$ & $\begin{array}{c}535.0 \\
532.8-537.2\end{array}$ & $137 \pm 1$ & $\begin{array}{c}136.7 \\
136.6-136.8\end{array}$ & $18.2 \pm 0.5$ & $\begin{array}{c}18.15 \\
17.97-18.33\end{array}$ & $692 \pm 12$ & $\begin{array}{c}691.9 \\
687.8-696.0\end{array}$ & $1929 \pm 2$ & $\begin{array}{c}1928.7 \\
1928.0-1929.4\end{array}$ \\
\hline 8 & $0.0145 \pm 0.0001$ & $\begin{array}{c}0.01451 \\
0.01452-0.01455\end{array}$ & $511 \pm 1$ & $\begin{array}{c}511.3 \\
511.0-511.6\end{array}$ & $156 \pm 1$ & $\begin{array}{c}156.1 \\
155.8-156.3\end{array}$ & $20.0 \pm 0.1$ & $\begin{array}{c}20.02 \\
19.98-20.06\end{array}$ & $582 \pm 13$ & $\begin{array}{c}581.8 \\
577.1-586.6\end{array}$ & $2395 \pm 12$ & $\begin{array}{c}2394.8 \\
2390.4-2399.2\end{array}$ \\
\hline 9 & $0.0116 \pm 0.0004$ & $\begin{array}{c}0.01157 \\
0.01144-0.01171\end{array}$ & $533 \pm 10$ & $\begin{array}{c}532.4 \\
529.0-535.9\end{array}$ & $197 \pm 4$ & $\begin{array}{c}196.5 \\
195.0-198.1\end{array}$ & $19.2 \pm 0.6$ & $\begin{array}{c}19.20 \\
18.98-19.42\end{array}$ & $865 \pm 14$ & $\begin{array}{c}865.1 \\
860.1-870.2\end{array}$ & $2607 \pm 56$ & $\begin{array}{c}2607.0 \\
2587.3-2626.8\end{array}$ \\
\hline 10 & $0.0045 \pm 0.0001$ & $\begin{array}{c}0.00451 \\
0.00448-0.00454\end{array}$ & $494 \pm 5$ & $\begin{array}{c}493.7 \\
491.9-495.5\end{array}$ & $154 \pm 2$ & $\begin{array}{c}153.5 \\
152.8-154.1\end{array}$ & $18.0 \pm 0.3$ & $\begin{array}{c}18.03 \\
17.94-18.12\end{array}$ & $632 \pm 11$ & $\begin{array}{c}631.6 \\
627.7-635.4\end{array}$ & $2410 \pm 42$ & $\begin{array}{c}2410.6 \\
2395.9-2425.3\end{array}$ \\
\hline 11 & $0.0033 \pm 0.0001$ & $\begin{array}{c}0.00333 \\
0.00332-0.00335\end{array}$ & $458 \pm 5$ & $\begin{array}{c}457.6 \\
455.9-459.2\end{array}$ & $129 \pm 2$ & $\begin{array}{c}128.5 \\
127.7-129.3\end{array}$ & $18.4 \pm 0.1$ & $\begin{array}{c}18.36 \\
18.34-18.38\end{array}$ & $759 \pm 13$ & $\begin{array}{c}758.9 \\
754.2-763.6\end{array}$ & $2028 \pm 26$ & $\begin{array}{c}2028.0 \\
2018.9-2037.2\end{array}$ \\
\hline
\end{tabular}

Table 3. Descriptive statistics of $\mathrm{Ca}, \mathrm{Ti}, \mathrm{Co}, \mathrm{Cu}, \mathrm{Ga}$ and $\mathrm{Rb}$ calculated on a total of 12 determinations for each formula and expressed as $\mu \mathrm{g} \mathrm{g}^{-1}$.

\begin{tabular}{|c|c|c|c|c|c|c|c|c|c|c|c|c|}
\hline \multirow{2}{*}{$\begin{array}{l}\text { Infant } \\
\text { Formula }\end{array}$} & \multicolumn{2}{|c|}{$\mathrm{Ca}$} & \multicolumn{2}{|c|}{$\mathrm{Ti}$} & \multicolumn{2}{|c|}{ Co } & \multicolumn{2}{|c|}{$\mathrm{Cu}$} & \multicolumn{2}{|c|}{ Ga } & \multicolumn{2}{|c|}{$\mathbf{R b}$} \\
\hline & $\mathbf{A M} \pm \mathbf{S D}$ & $\begin{array}{l}\text { Median } \\
\text { IQR }\end{array}$ & $\mathbf{A M} \pm \mathbf{S D}$ & $\begin{array}{l}\text { Median } \\
\text { IQR }\end{array}$ & $\mathbf{A M} \pm \mathbf{S D}$ & $\begin{array}{c}\text { Median } \\
\text { IQR }\end{array}$ & $\mathbf{A M} \pm \mathbf{S D}$ & $\begin{array}{c}\text { Median } \\
\text { IQR }\end{array}$ & $\mathbf{A M} \pm \mathbf{S D}$ & $\begin{array}{l}\text { Median } \\
\text { IQR }\end{array}$ & $\mathbf{A M} \pm \mathbf{S D}$ & $\begin{array}{c}\text { Median } \\
\text { IQR }\end{array}$ \\
\hline 1 & $2729 \pm 36$ & $\begin{array}{c}2729.0 \\
2716.3-2741.8\end{array}$ & $0.105 \pm 0.005$ & $\begin{array}{c}0.1054 \\
0.1035-0.1073\end{array}$ & $0.012 \pm 0.001$ & $\begin{array}{c}0.0118 \\
0.0115-0.0120\end{array}$ & $1.33 \pm 0.08$ & $\begin{array}{c}1.329 \\
1.299-1.358\end{array}$ & $\begin{array}{c}0.004 \pm \\
0.001\end{array}$ & $\begin{array}{c}0.0036 \\
0.0033-0.0038\end{array}$ & $2.92 \pm 0.05$ & $\begin{array}{c}2.922 \\
2.906-2.939\end{array}$ \\
\hline 2 & $2144 \pm 14$ & $\begin{array}{c}2143.9 \\
2138.8-2148.9\end{array}$ & $0.098 \pm 0.001$ & $\begin{array}{c}0.0977 \\
0.0974-0.0979\end{array}$ & $0.010 \pm 0.001$ & $\begin{array}{c}0.0097 \\
0.0096-0.0097\end{array}$ & $\begin{array}{c}1.731 \pm \\
0.001\end{array}$ & $\begin{array}{c}1.731 \\
1.730-1.732\end{array}$ & $\begin{array}{c}0.003 \pm \\
0.001\end{array}$ & $\begin{array}{c}0.0027 \\
0.0025-0.0029\end{array}$ & $2.48 \pm 0.10$ & $\begin{array}{c}2.482 \\
2.448-2.517\end{array}$ \\
\hline 3 & $2687 \pm 16$ & $\begin{array}{c}2686.5 \\
2680.8-2692.1\end{array}$ & $0.092 \pm 0.006$ & $\begin{array}{c}0.0922 \\
0.0903-0.0942\end{array}$ & $0.014 \pm 0.001$ & $\begin{array}{c}0.0144 \\
0.0142-0.0146\end{array}$ & $1.04 \pm 0.03$ & $\begin{array}{c}1.036 \\
1.027-1.046\end{array}$ & $\begin{array}{c}0.003 \pm \\
0.001\end{array}$ & $\begin{array}{c}0.0032 \\
0.0029-0.0035\end{array}$ & $2.93 \pm 0.01$ & $\begin{array}{c}2.930 \\
2.928-2.933\end{array}$ \\
\hline
\end{tabular}


Table 3. Cont.

\begin{tabular}{|c|c|c|c|c|c|c|c|c|c|c|c|c|}
\hline \multirow[b]{2}{*}{$\begin{array}{l}\text { Infant } \\
\text { Formula }\end{array}$} & \multicolumn{2}{|c|}{$\mathrm{Ca}$} & \multicolumn{2}{|c|}{$\mathrm{Ti}$} & \multicolumn{2}{|c|}{ Co } & \multicolumn{2}{|c|}{$\mathrm{Cu}$} & \multicolumn{2}{|c|}{$\mathrm{Ga}$} & \multicolumn{2}{|c|}{$\mathbf{R} \mathbf{b}$} \\
\hline & $\mathbf{A M} \pm \mathbf{S D}$ & $\begin{array}{l}\text { Median } \\
\text { IQR }\end{array}$ & $\mathbf{A M} \pm \mathbf{S D}$ & $\begin{array}{l}\text { Median } \\
\text { IQR }\end{array}$ & $\mathbf{A M} \pm \mathbf{S D}$ & $\begin{array}{l}\text { Median } \\
\text { IQR }\end{array}$ & $\mathbf{A M} \pm \mathbf{S D}$ & $\begin{array}{l}\text { Median } \\
\text { IQR }\end{array}$ & $\mathbf{A M} \pm \mathbf{S D}$ & $\begin{array}{l}\text { Median } \\
\text { IQR }\end{array}$ & $\mathbf{A M} \pm \mathbf{S D}$ & $\begin{array}{l}\text { Median } \\
\text { IQR }\end{array}$ \\
\hline 4 & $2119 \pm 31$ & $\begin{array}{c}2118.6 \\
2107.5-2129.6\end{array}$ & $0.089 \pm 0.001$ & $\begin{array}{c}0.0893 \\
0.0890-0.0896\end{array}$ & $0.011 \pm 0.001$ & $\begin{array}{c}0.0113 \\
0.0112-0.0115\end{array}$ & $1.40 \pm 0.05$ & $\begin{array}{c}1.395 \\
1.377-1.413\end{array}$ & $\begin{array}{c}0.005 \pm \\
0.002\end{array}$ & $\begin{array}{c}0.0047 \\
0.0040-0.0054\end{array}$ & $2.51 \pm 0.57$ & $\begin{array}{c}2.510 \\
2.309-2.711\end{array}$ \\
\hline 5 & $1783 \pm 20$ & $\begin{array}{c}1783.3 \\
1776.2-1790.4\end{array}$ & $0.112 \pm 0.013$ & $\begin{array}{c}0.1125 \\
0.1080-0.1170\end{array}$ & $0.008 \pm 0.001$ & $\begin{array}{c}0.0081 \\
0.0079-0.0083\end{array}$ & $1.64 \pm 0.20$ & $\begin{array}{c}1.643 \\
1.574-1.713\end{array}$ & $\begin{array}{c}0.003 \pm \\
0.001\end{array}$ & $\begin{array}{c}0.0027 \\
0.0026-0.0028\end{array}$ & $0.60 \pm 0.02$ & $\begin{array}{c}0.595 \\
0.590-0.601\end{array}$ \\
\hline 6 & $2517 \pm 41$ & $\begin{array}{c}2516.9 \\
2502.3-2531.5\end{array}$ & $0.099 \pm 0.002$ & $\begin{array}{c}0.0991 \\
0.0983-0.0998\end{array}$ & $0.010 \pm 0.001$ & $\begin{array}{c}0.0104 \\
0.0104-0.0105\end{array}$ & $1.05 \pm 0.03$ & $\begin{array}{c}1.047 \\
1.038-1.056\end{array}$ & $\begin{array}{c}0.003 \pm \\
0.001\end{array}$ & $\begin{array}{c}0.0031 \\
0.0030-0.0032\end{array}$ & $2.22 \pm 0.13$ & $\begin{array}{c}2.217 \\
2.172-2.263\end{array}$ \\
\hline 7 & $2423 \pm 56$ & $\begin{array}{c}2423.2 \\
2403.6-2442.9\end{array}$ & $0.092 \pm 0.004$ & $\begin{array}{c}0.0920 \\
0.0907-0.0933\end{array}$ & $0.010 \pm 0.001$ & $\begin{array}{c}0.0096 \\
0.0095-0.0097\end{array}$ & $\begin{array}{c}0.800 \pm \\
0.008\end{array}$ & $\begin{array}{c}0.800 \\
0.797-0.803\end{array}$ & $\begin{array}{c}0.003 \pm \\
0.001\end{array}$ & $\begin{array}{c}0.0033 \\
0.0032-0.0034\end{array}$ & $2.26 \pm 0.16$ & $\begin{array}{c}2.259 \\
2.204-2.314\end{array}$ \\
\hline 8 & $2478 \pm 2$ & $\begin{array}{c}2477.5 \\
2476.9-2478.2\end{array}$ & $0.122 \pm 0.004$ & $\begin{array}{c}0.1217 \\
0.1202-0.1231\end{array}$ & $0.010 \pm 0.001$ & $\begin{array}{c}0.0100 \\
0.0099-0.0101\end{array}$ & $1.11 \pm 0.01$ & $\begin{array}{c}1.111 \\
1.107-1.116\end{array}$ & $\begin{array}{c}0.002 \pm \\
0.001\end{array}$ & $\begin{array}{c}0.0016 \\
0.0015-0.0018\end{array}$ & $0.39 \pm 0.01$ & $\begin{array}{c}0.386 \\
0.383-0.389\end{array}$ \\
\hline 9 & $2814 \pm 17$ & $\begin{array}{c}2813.6 \\
2807.6-2819.5\end{array}$ & $0.103 \pm 0.004$ & $\begin{array}{c}0.1028 \\
0.1014-0.1043\end{array}$ & $0.013 \pm 0.001$ & $\begin{array}{c}0.0133 \\
0.0132-0.0134\end{array}$ & $1.35 \pm 0.03$ & $\begin{array}{c}1.351 \\
1.339-1.364\end{array}$ & $\begin{array}{c}0.007 \pm \\
0.001\end{array}$ & $\begin{array}{c}0.0068 \\
0.0066-0.0071\end{array}$ & $7.27 \pm 0.18$ & $\begin{array}{c}7.268 \\
7.204-7.332\end{array}$ \\
\hline 10 & $2303 \pm 28$ & $\begin{array}{c}2302.5 \\
2292.5-2312.5\end{array}$ & $0.081 \pm 0.002$ & $\begin{array}{c}0.0815 \\
0.0807-0.0823\end{array}$ & $0.009 \pm 0.001$ & $\begin{array}{c}0.0093 \\
0.0092-0.0093\end{array}$ & $1.13 \pm 0.03$ & $\begin{array}{c}1.131 \\
1.122-1.141\end{array}$ & $\begin{array}{c}0.004 \pm \\
0.001\end{array}$ & $\begin{array}{c}0.0043 \\
0.0041-0.0044\end{array}$ & $4.02 \pm 0.10$ & $\begin{array}{c}4.016 \\
3.980-4.052\end{array}$ \\
\hline 11 & $2505 \pm 22$ & $\begin{array}{c}2505.3 \\
2497.6-2512.9\end{array}$ & $0.084 \pm 0.002$ & $\begin{array}{c}0.0836 \\
0.0830-0.0843\end{array}$ & $0.014 \pm 0.001$ & $\begin{array}{c}0.0143 \\
0.0140-0.0146\end{array}$ & $1.13 \pm 0.02$ & $\begin{array}{c}1.126 \\
1.119-1.133\end{array}$ & $\begin{array}{c}0.004 \pm \\
0.001\end{array}$ & $\begin{array}{c}0.0038 \\
0.0035-0.0040\end{array}$ & $3.97 \pm 0.20$ & $\begin{array}{c}3.968 \\
3.896-4.040\end{array}$ \\
\hline
\end{tabular}

$\mathrm{AM}=$ arithmetic mean; $\mathrm{SD}=$ standard deviation; $\mathrm{IQR}=$ interquartile range.

Table 4. Descriptive statistics of Sr, Mo, Sn, Cs, Ba and La calculated on a total of 12 determinations for each formula and expressed as $\mu g \mathrm{~g}^{-1}$.

\begin{tabular}{|c|c|c|c|c|c|c|c|c|c|c|c|c|}
\hline \multirow[b]{2}{*}{$\begin{array}{l}\text { Infant } \\
\text { Formula }\end{array}$} & \multicolumn{2}{|c|}{$\mathrm{Sr}$} & \multicolumn{2}{|c|}{ Mo } & \multicolumn{2}{|c|}{ Sn } & \multicolumn{2}{|c|}{ Cs } & \multicolumn{2}{|c|}{$\mathrm{Ba}$} & \multicolumn{2}{|c|}{$\mathrm{La}$} \\
\hline & $\mathbf{A M} \pm \mathbf{S D}$ & $\begin{array}{c}\text { Median } \\
\text { IQR }\end{array}$ & $\mathbf{A M} \pm \mathbf{S D}$ & $\begin{array}{c}\text { Median } \\
\text { IQR }\end{array}$ & $\mathbf{A M} \pm \mathbf{S D}$ & $\begin{array}{c}\text { Median } \\
\text { IQR }\end{array}$ & $\mathrm{AM} \pm \mathrm{SD}$ & $\begin{array}{c}\text { Median } \\
\text { IQR }\end{array}$ & $\mathbf{A M} \pm \mathbf{S D}$ & $\begin{array}{c}\text { Median } \\
\text { IQR }\end{array}$ & $\mathbf{A M} \pm \mathbf{S D}$ & $\begin{array}{c}\text { Median } \\
\text { IQR }\end{array}$ \\
\hline 1 & $1.59 \pm 0.02$ & $\begin{array}{c}1.590 \\
1.583-1.598\end{array}$ & $0.172 \pm 0.002$ & $\begin{array}{c}0.1717 \\
0.1709-0.1724\end{array}$ & $0.0969 \pm 0.0874$ & $\begin{array}{c}0.09694 \\
0.06603-0.12785\end{array}$ & $0.0190 \pm 0.0009$ & $\begin{array}{c}0.01898 \\
0.01865-0.01931\end{array}$ & $0.214 \pm 0.001$ & $\begin{array}{c}0.2138 \\
0.2136-0.2140\end{array}$ & $0.0063 \pm 0.0044$ & $\begin{array}{c}0.00632 \\
0.00476-0.00789\end{array}$ \\
\hline 3. & $1.02 \pm 0.01$ & $\begin{array}{c}1.019 \\
1.017-1.021\end{array}$ & $0.189 \pm 0.006$ & $\begin{array}{c}0.1886 \\
0.1867-0.1906\end{array}$ & $0.0028 \pm 0.0012$ & $\begin{array}{c}0.00282 \\
0.00239-0.00326\end{array}$ & $0.0038 \pm 0.0001$ & $\begin{array}{c}0.00382 \\
0.00381-0.00383\end{array}$ & $0.121 \pm 0.008$ & $\begin{array}{c}0.1390-0.1419 \\
0.1205 \\
0.1178-0.1232\end{array}$ & $0.0011 \pm 0.0001$ & $\begin{array}{c}0.00285-0.00290 \\
0.00107 \\
0.00105-0.00108\end{array}$ \\
\hline 4 & $1.12 \pm 0.24$ & $\begin{array}{c}1.115 \\
1.029-1.201\end{array}$ & $0.107 \pm 0.017$ & $\begin{array}{c}0.1075 \\
0.1014-0.1135\end{array}$ & $0.0106 \pm 0.0038$ & $\begin{array}{c}0.01058 \\
0.00922-0.01194\end{array}$ & $0.0052 \pm 0.0002$ & $\begin{array}{c}0.00519 \\
0.00512-0.00525\end{array}$ & $0.207 \pm 0.021$ & $\begin{array}{c}0.2073 \\
0.1998-0.2149\end{array}$ & $0.0036 \pm 0.0001$ & $\begin{array}{c}0.00356 \\
0.00354-0.00358\end{array}$ \\
\hline 5 & $0.529 \pm 0.018$ & $\begin{array}{c}0.529 \\
0.523-0.536\end{array}$ & $0.115 \pm 0.001$ & $\begin{array}{c}0.1149 \\
0.1145-0.1154\end{array}$ & $0.0237 \pm 0.0051$ & $\begin{array}{c}0.02371 \\
0.02190-0.02551\end{array}$ & $0.0015 \pm 0.0001$ & $\begin{array}{c}0.00151 \\
0.00147-0.00154\end{array}$ & $0.111 \pm 0.011$ & $\begin{array}{c}0.1109 \\
0.1069-0.1148\end{array}$ & $0.0006 \pm 0.0002$ & $\begin{array}{c}0.00061 \\
0.00055-0.00068\end{array}$ \\
\hline 6 & $1.44 \pm 0.11$ & $\begin{array}{c}1.438 \\
1.401-1.475\end{array}$ & $0.242 \pm 0.018$ & $\begin{array}{c}0.2422 \\
0.2360-0.2484\end{array}$ & $0.0032 \pm 0.0007$ & $\begin{array}{c}0.00319 \\
0.00294-0.00345\end{array}$ & $0.0074 \pm 0.0002$ & $\begin{array}{c}0.00745 \\
0.00736-0.00754\end{array}$ & $0.140 \pm 0.002$ & $\begin{array}{c}0.1397 \\
0.1391-0.1402\end{array}$ & $0.0002 \pm 0.0001$ & $\begin{array}{c}0.00025 \\
0.00023-0.00026\end{array}$ \\
\hline
\end{tabular}


Table 4. Cont.

\begin{tabular}{|c|c|c|c|c|c|c|c|c|c|c|c|c|}
\hline \multirow[b]{2}{*}{$\begin{array}{l}\text { Infant - } \\
\text { Formula }\end{array}$} & \multicolumn{2}{|c|}{$\mathrm{Sr}$} & \multicolumn{2}{|c|}{ Mo } & \multicolumn{2}{|c|}{ Sn } & \multicolumn{2}{|c|}{ Cs } & \multicolumn{2}{|c|}{ Ba } & \multicolumn{2}{|c|}{ La } \\
\hline & $\mathrm{AM} \pm \mathbf{S D}$ & $\begin{array}{c}\text { Median } \\
\text { IQR }\end{array}$ & $\mathbf{A M} \pm \mathbf{S D}$ & $\begin{array}{c}\text { Median } \\
\text { IQR }\end{array}$ & $\mathbf{A M} \pm \mathbf{S D}$ & $\begin{array}{c}\text { Median } \\
\text { IQR }\end{array}$ & $\mathrm{AM} \pm \mathrm{SD}$ & $\begin{array}{c}\text { Median } \\
\text { IQR }\end{array}$ & $\mathrm{AM} \pm \mathrm{SD}$ & $\begin{array}{c}\text { Median } \\
\text { IQR }\end{array}$ & $\mathrm{AM} \pm \mathrm{SD}$ & $\begin{array}{c}\text { Median } \\
\text { IQR }\end{array}$ \\
\hline 7 & $1.44 \pm 0.12$ & $\begin{array}{c}1.440 \\
1.397-1.482\end{array}$ & $0.276 \pm 0.037$ & $\begin{array}{c}0.2763 \\
0.2633-0.2893\end{array}$ & $0.0054 \pm 0.0049$ & $\begin{array}{c}0.00539 \\
0.00367-0.00712\end{array}$ & $0.0079 \pm 0.0003$ & $\begin{array}{c}0.00794 \\
0.00783-0.00805\end{array}$ & $0.179 \pm 0.018$ & $\begin{array}{c}0.1788 \\
0.1725-0.1851\end{array}$ & $0.0010 \pm 0.0002$ & $\begin{array}{c}0.00097 \\
0.00091-0.00103\end{array}$ \\
\hline 8 & $0.827 \pm 0.015$ & $\begin{array}{c}0.827 \\
0.822-0.833\end{array}$ & $0.056 \pm 0.004$ & $\begin{array}{c}0.0564 \\
0.0551-0.0578\end{array}$ & $0.0009 \pm 0.0001$ & $\begin{array}{c}0.00094 \\
0.00093-0.00096\end{array}$ & $0.0057 \pm 0.0003$ & $\begin{array}{c}0.00568 \\
0.00558-0.00578\end{array}$ & $0.074 \pm 0.002$ & $\begin{array}{c}0.0739 \\
0.0730-0.0747\end{array}$ & $0.0211 \pm 0.0002$ & $\begin{array}{c}0.02110 \\
0.02104-0.02117\end{array}$ \\
\hline 9 & $2.34 \pm 0.04$ & $\begin{array}{c}2.340 \\
2.326-2.354\end{array}$ & $0.124 \pm 0.005$ & $\begin{array}{c}0.1235 \\
0.1217-0.1254\end{array}$ & $0.0003 \pm 0.0001$ & $\begin{array}{c}0.00029 \\
0.00028-0.00030\end{array}$ & $0.0145 \pm 0.0003$ & $\begin{array}{c}0.01455 \\
0.01443-0.01466\end{array}$ & $0.420 \pm 0.003$ & $\begin{array}{c}0.4198 \\
0.4188-0.4208\end{array}$ & $0.0018 \pm 0.0001$ & $\begin{array}{c}0.00185 \\
0.00181-0.00189\end{array}$ \\
\hline 10 & $1.08 \pm 0.01$ & $\begin{array}{c}1.077 \\
1.073-1.081\end{array}$ & $0.195 \pm 0.002$ & $\begin{array}{c}0.1948 \\
0.1940-0.1955\end{array}$ & $0.0007 \pm 0.0001$ & $\begin{array}{c}0.00071 \\
0.00070-0.00072\end{array}$ & $0.0127 \pm 0.0002$ & $\begin{array}{c}0.01274 \\
0.01269-0.01280\end{array}$ & $0.180 \pm 0.001$ & $\begin{array}{c}0.1805 \\
0.1800-0.1810\end{array}$ & $0.0040 \pm 0.0001$ & $\begin{array}{c}0.00401 \\
0.00400-0.00402\end{array}$ \\
\hline 11 & $0.78 \pm 0.04$ & $\begin{array}{c}0.781 \\
0.767-0.796\end{array}$ & $0.146 \pm 0.003$ & $\begin{array}{c}0.1458 \\
0.1446-0.1470\end{array}$ & $0.0269 \pm 0.0003$ & $\begin{array}{c}0.02688 \\
0.02677-0.02700\end{array}$ & $0.0043 \pm 0.0002$ & $\begin{array}{c}0.00434 \\
0.00428-0.00440\end{array}$ & $0.137 \pm 0.002$ & $\begin{array}{c}0.1369 \\
0.1361-0.1377\end{array}$ & $0.0022 \pm 0.0001$ & $\begin{array}{c}0.00216 \\
0.00213-0.00219\end{array}$ \\
\hline
\end{tabular}

$\mathrm{AM}=$ arithmetic mean; $\mathrm{SD}=$ standard deviation; $\mathrm{IQR}=$ interquartile range.

Table 5. Descriptive statistics of Ce, $\mathrm{Tl}$, and Bi calculated on a total of 12 determinations for each formula and expressed as $\mu \mathrm{g} \mathrm{g}^{-1}$.

\begin{tabular}{|c|c|c|c|c|c|c|}
\hline \multirow{2}{*}{$\begin{array}{c}\text { Infant } \\
\text { Formula }\end{array}$} & \multicolumn{2}{|c|}{$\mathrm{Ce}$} & \multicolumn{2}{|c|}{ T1 } & \multicolumn{2}{|c|}{$\mathbf{B i}$} \\
\hline & $\mathbf{A M} \pm \mathbf{S D}$ & $\begin{array}{l}\text { Median } \\
\text { IQR }\end{array}$ & $\mathbf{A M} \pm \mathbf{S D}$ & $\begin{array}{l}\text { Median } \\
\text { IQR }\end{array}$ & $\mathbf{A M} \pm \mathbf{S D}$ & $\begin{array}{l}\text { Median } \\
\text { IQR }\end{array}$ \\
\hline 1 & $0.0056 \pm 0.0054$ & $\begin{array}{c}0.00560 \\
0.00368-0.00753\end{array}$ & $0.00042 \pm 0.00006$ & $\begin{array}{c}0.000418 \\
0.000394-0.000441\end{array}$ & $0.0040 \pm 0.0005$ & $\begin{array}{c}0.00404 \\
0.00388-0.00420\end{array}$ \\
\hline 2 & $0.0011 \pm 0.0001$ & $\begin{array}{c}0.00114 \\
0.00111-0.00116\end{array}$ & $0.00069 \pm 0.00001$ & $\begin{array}{c}0.000685 \\
0.000682-0.000688\end{array}$ & $0.0035 \pm 0.0002$ & $\begin{array}{c}0.00347 \\
0.00340-0.00353\end{array}$ \\
\hline 3. & $0.0006 \pm 0.0001$ & $\begin{array}{c}0.00063 \\
0.00061-0.00065\end{array}$ & $0.00061 \pm 0.00004$ & $\begin{array}{c}0.000606 \\
0.000589-0.000623\end{array}$ & $0.0014 \pm 0.0001$ & $\begin{array}{c}0.00141 \\
0.00127-0.00144\end{array}$ \\
\hline 4 & $0.0013 \pm 0.0001$ & $\begin{array}{c}0.00129 \\
0.00126-0.00133\end{array}$ & $0.00020 \pm 0.00012$ & $\begin{array}{c}0.000199 \\
0.000155-0.000242\end{array}$ & $0.0013 \pm 0.0001$ & $\begin{array}{c}0.00138 \\
0.00126-0.00127\end{array}$ \\
\hline 5 & $0.0009 \pm 0.0001$ & $\begin{array}{c}0.00093 \\
0.00091-0.00095\end{array}$ & $0.00084 \pm 0.00007$ & $\begin{array}{c}0.000838 \\
0.000812-0.000863\end{array}$ & $0.0004 \pm 0.0001$ & $\begin{array}{c}0.00042 \\
0.00042-0.00042\end{array}$ \\
\hline 6 & $0.0003 \pm 0.0001$ & $\begin{array}{c}0.00026 \\
0.00026-0.00027\end{array}$ & $0.00055 \pm 0.00013$ & $\begin{array}{c}0.000551 \\
0.000505-0.000598\end{array}$ & $0.0023 \pm 0.0003$ & $\begin{array}{c}0.00231 \\
0.00218-0.00243\end{array}$ \\
\hline 7 & $0.0009 \pm 0.0001$ & $\begin{array}{c}0.00086 \\
0.00085-0.00087\end{array}$ & $0.00054 \pm 0.00004$ & $\begin{array}{c}0.000544 \\
0.000529-0.000559\end{array}$ & $0.0022 \pm 0.0002$ & $\begin{array}{c}0.00219 \\
0.00212-0.00225\end{array}$ \\
\hline 8 & $0.0067 \pm 0.0003$ & $\begin{array}{c}0.00670 \\
0.00659-0.00681\end{array}$ & $0.00109 \pm 0.00004$ & $\begin{array}{c}0.001086 \\
0.001071-0.001101\end{array}$ & $0.0123 \pm 0.0001$ & $\begin{array}{c}0.01227 \\
0.01224-0.01230\end{array}$ \\
\hline
\end{tabular}


Table 5. Cont.

\begin{tabular}{|c|c|c|c|c|c|c|}
\hline \multirow{2}{*}{$\begin{array}{l}\text { Infant } \\
\text { Formula }\end{array}$} & \multicolumn{2}{|c|}{$\mathrm{Ce}$} & \multicolumn{2}{|c|}{$\mathrm{Tl}$} & \multicolumn{2}{|c|}{$\mathbf{B i}$} \\
\hline & $\mathbf{A M} \pm \mathbf{S D}$ & $\begin{array}{l}\text { Median } \\
\text { IQR }\end{array}$ & $\mathrm{AM} \pm \mathrm{SD}$ & $\begin{array}{l}\text { Median } \\
\text { IQR }\end{array}$ & $\mathbf{A M} \pm \mathbf{S D}$ & $\begin{array}{l}\text { Median } \\
\text { IQR }\end{array}$ \\
\hline 9 & $0.0010 \pm 0.0002$ & $\begin{array}{c}0.00101 \\
0.00094-0.00107\end{array}$ & $0.00038 \pm 0.00001$ & $\begin{array}{c}0.000383 \\
0.000377-0.000389\end{array}$ & $0.0004 \pm 0.0001$ & $\begin{array}{c}0.00042 \\
0.00042-0.00042\end{array}$ \\
\hline 10 & $0.0017 \pm 0.0001$ & $\begin{array}{c}0.00172 \\
0.00167-0.00176\end{array}$ & $0.00131 \pm 0.00007$ & $\begin{array}{c}0.001308 \\
0.001280-0.001336\end{array}$ & $0.0017 \pm 0.0001$ & $\begin{array}{c}0.00171 \\
0.00171-0.00172\end{array}$ \\
\hline 11 & $0.0010 \pm 0.0001$ & $\begin{array}{c}0.00101 \\
0.00099-0.00104\end{array}$ & $0.00055 \pm 0.00001$ & $\begin{array}{c}0.000550 \\
0.000549-0.000551\end{array}$ & $0.0008 \pm 0.0005$ & $\begin{array}{c}0.00076- \\
0.00059-0.00093\end{array}$ \\
\hline
\end{tabular}

$\mathrm{AM}=$ arithmetic mean; $\mathrm{SD}$ = standard deviation; $\mathrm{IQR}=$ interquartile range.

Table 6. Descriptive statistics of $\mathrm{U}$, Fe and Se calculated on a total of 12 determinations for each formula and expressed as $\mu \mathrm{g} \mathrm{g}^{-1}$.

\begin{tabular}{|c|c|c|c|c|c|c|}
\hline \multirow{2}{*}{$\begin{array}{l}\text { Infant } \\
\text { Formula }\end{array}$} & \multicolumn{2}{|c|}{$\mathbf{U}$} & \multicolumn{2}{|c|}{$\mathrm{Fe}$} & \multicolumn{2}{|c|}{$\mathrm{Se}$} \\
\hline & $\mathrm{AM} \pm \mathrm{SD}$ & $\begin{array}{l}\text { Median } \\
\text { IQR }\end{array}$ & $\mathrm{AM} \pm \mathrm{SD}$ & $\begin{array}{l}\text { Median } \\
\text { IQR }\end{array}$ & $\mathbf{A M} \pm \mathbf{S D}$ & $\begin{array}{l}\text { Median } \\
\text { IQR }\end{array}$ \\
\hline 1 & $0.00074 \pm 0.00001$ & $\begin{array}{c}0.000740 \\
0.000739-0.000741\end{array}$ & $31.4 \pm 0.3$ & $\begin{array}{c}31.40 \\
31.28-31.52\end{array}$ & $0.151 \pm 0.007$ & $\begin{array}{c}0.1513 \\
0.14893-0.15359\end{array}$ \\
\hline 2 & $0.00068 \pm 0.00001$ & $\begin{array}{c}0.000681 \\
0.000675-0.000687\end{array}$ & $32.2 \pm 0.7$ & $\begin{array}{c}32.21 \\
31.96-32.46\end{array}$ & $0.143 \pm 0.014$ & $\begin{array}{c}0.1431 \\
0.13824-0.14787\end{array}$ \\
\hline 3 & $0.00127 \pm 0.00012$ & $\begin{array}{c}0.001272 \\
0.001231-0.001312\end{array}$ & $28.6 \pm 0.2$ & $\begin{array}{c}28.62 \\
28.56-28.67\end{array}$ & $0.147 \pm 0.018$ & $\begin{array}{c}0.1468 \\
0.14048-0.15304\end{array}$ \\
\hline 4 & $0.00032 \pm 0.00001$ & $\begin{array}{c}0.000318 \\
0.000305-0.000331\end{array}$ & $32.9 \pm 0.2$ & $\begin{array}{c}32.88 \\
32.80-32.96\end{array}$ & $0.088 \pm 0.005$ & $\begin{array}{c}0.0885 \\
0.08680-0.09018\end{array}$ \\
\hline 5 & $0.00385 \pm 0.00001$ & $\begin{array}{c}0.003854 \\
0.003850-0.003858\end{array}$ & $28.1 \pm 0.6$ & $\begin{array}{c}28.11 \\
27.90-28.31\end{array}$ & $0.198 \pm 0.0010$ & $\begin{array}{c}0.1980 \\
0.19763-0.19834\end{array}$ \\
\hline 6 & $0.00053 \pm 0.00001$ & $\begin{array}{c}0.000530 \\
0.000516-0.000545\end{array}$ & $27.0 \pm 0.3$ & $\begin{array}{c}27.00 \\
26.90-27.11\end{array}$ & $0.139 \pm 0.024$ & $\begin{array}{c}0.1390 \\
0.13034-0.14758\end{array}$ \\
\hline 7 & $0.00159 \pm 0.00111$ & $\begin{array}{c}0.001585 \\
0.001193-0.001978\end{array}$ & $23.9 \pm 1.4$ & $\begin{array}{c}23.98 \\
23.49-24.48\end{array}$ & $0.111 \pm 0.029$ & $\begin{array}{c}0.1106 \\
0.10043-0.12069\end{array}$ \\
\hline 8 & $0.00566 \pm 0.00020$ & $\begin{array}{c}0.005657 \\
0.005585-0.005730\end{array}$ & $24.3 \pm 0.1$ & $\begin{array}{c}24.23 \\
24.18-24.28\end{array}$ & $0.158 \pm 0.026$ & $\begin{array}{c}0.1576 \\
0.14834-0.16679\end{array}$ \\
\hline
\end{tabular}


Table 6. Cont

\begin{tabular}{|c|c|c|c|c|c|c|}
\hline \multirow{2}{*}{$\begin{array}{c}\text { Infant } \\
\text { Formula }\end{array}$} & \multicolumn{2}{|c|}{$\mathrm{U}$} & \multicolumn{2}{|c|}{$\mathrm{Fe}$} & \multicolumn{2}{|c|}{$\mathrm{Se}$} \\
\hline & $\mathbf{A M} \pm \mathbf{S D}$ & $\begin{array}{c}\text { Median } \\
\text { IQR }\end{array}$ & $\mathrm{AM} \pm \mathrm{SD}$ & $\begin{array}{c}\text { Median } \\
\text { IQR }\end{array}$ & $\mathbf{A M} \pm \mathbf{S D}$ & $\begin{array}{c}\text { Median } \\
\text { IQR }\end{array}$ \\
\hline 10 & $0.00490 \pm 0.00029$ & $\begin{array}{c}0.004904 \\
0.004802-0.005007\end{array}$ & $21.6 \pm 0.6$ & $\begin{array}{c}21.63 \\
21.44-21.83\end{array}$ & $0.130 \pm 0.004$ & $\begin{array}{c}0.1304 \\
0.12902-0.13178\end{array}$ \\
\hline 11 & $0.00084 \pm 0.00001$ & $\begin{array}{c}0.000843 \\
0.000842-0.000844\end{array}$ & $34.0 \pm 0.3$ & $\begin{array}{c}33.96 \\
33.85-34.06\end{array}$ & $0.159 \pm 0.005$ & $\begin{array}{c}0.1591 \\
0.15737-0.16079\end{array}$ \\
\hline
\end{tabular}

$\mathrm{AM}=$ arithmetic mean; $\mathrm{SD}=$ standard deviation; $\mathrm{IQR}=$ interquartile range.

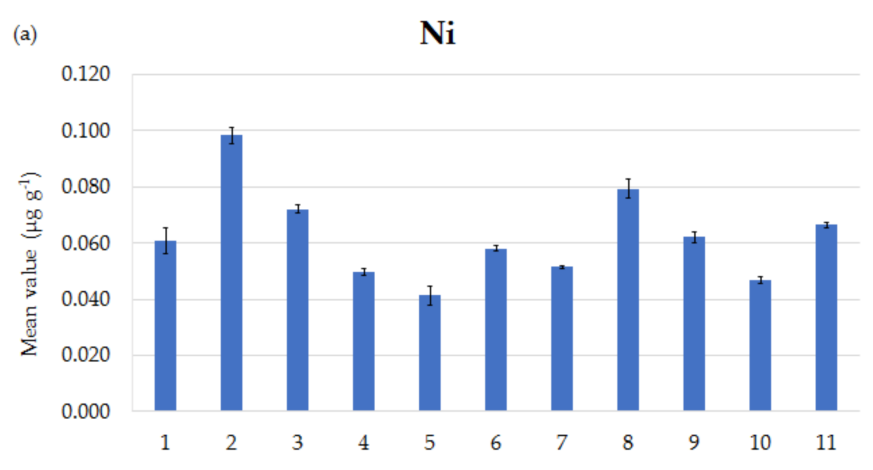

(c) 0.007

Cd

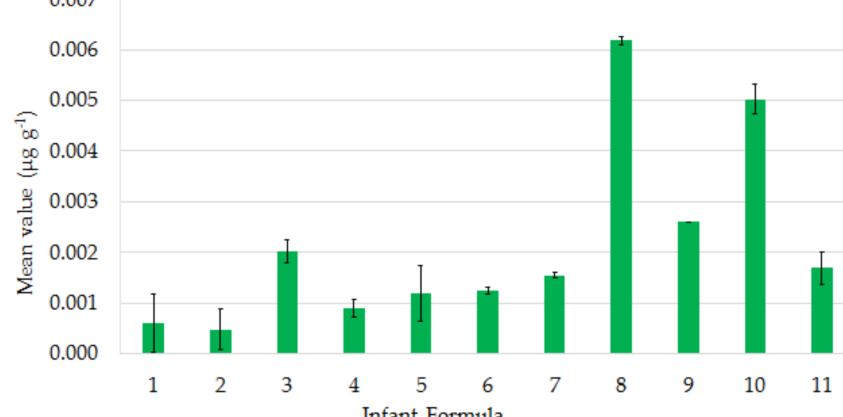

(b) 25.0

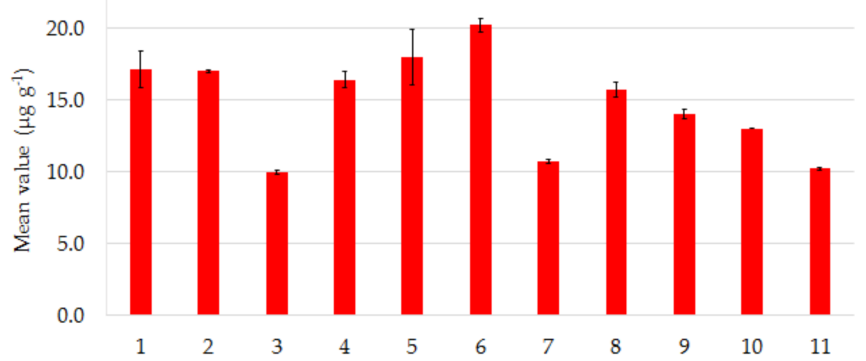

(d)

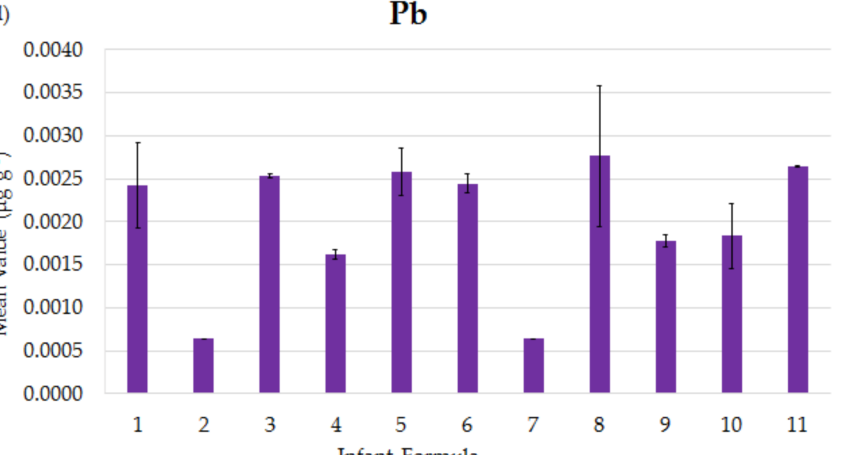

Figure 1. Mean values of the concentration, expressed in $\mu \mathrm{g} \mathrm{g}^{-1}$ for each formula, of (a) Ni, (b) $\mathrm{Zi}$, (c) Cd and (d) Pb. The black line on each bar represents the standard deviation. 
The highest levels of the selected elements were detected in formula 2 for $\mathrm{Ni}(0.098 \pm$ $\left.0.003 \mu \mathrm{g} \mathrm{g}^{-1}\right)$, in formula 6 for $\mathrm{Zn}\left(20.2 \pm 0.5 \mu \mathrm{g} \mathrm{g}^{-1}\right)$, and in formula 8 for $\mathrm{Cd}$ and $\mathrm{Pb}$ (respectively $0.0062 \pm 0.0001 \mu \mathrm{g} \mathrm{g}^{-1}$ and $0.0028 \pm 0.0008 \mu \mathrm{g} \mathrm{g}^{-1}$ ).

The concentrations of the daily/weekly intake of $\mathrm{Cd}, \mathrm{Ni}, \mathrm{Pb}$, and $\mathrm{Zn}$, calculated separately for males and females, are reported in Table 7.

Table 7. Daily intake of $\mathrm{Ni}$ and $\mathrm{Zn}$ and weekly intake of $\mathrm{Cd}$ and $\mathrm{Pb}$ for infants from 0 to 6 months, separately for males (M) and females $(\mathrm{F})$.

\begin{tabular}{|c|c|c|c|c|c|c|c|c|c|c|c|}
\hline \multirow[t]{2}{*}{ Age } & \multirow{2}{*}{$\begin{array}{l}\text { Amount of Infant } \\
\text { Formula } \\
\text { (g/Day } \\
\text { g/Week) }\end{array}$} & \multicolumn{2}{|c|}{ Weight (kg) } & \multicolumn{2}{|c|}{$\begin{array}{c}\quad \mathrm{Ni} \\
\mu \mathrm{g} \mathrm{kg} \mathrm{kg}^{-1} \\
\text { bw/Day }\end{array}$} & \multicolumn{2}{|c|}{$\begin{array}{l}\text { Zn } \\
\mu \mathrm{g} \mathrm{kg}^{-1} \\
\text { bw/Day }\end{array}$} & \multicolumn{2}{|c|}{$\begin{array}{c}\text { Cd } \\
\mu \mathrm{g} \mathrm{kg}^{-1} \\
\text { bw/Week }\end{array}$} & \multicolumn{2}{|c|}{$\begin{array}{c}\mathrm{Pb} \\
\mu \mathrm{g} \mathrm{kg}^{-1} \\
\text { bw/Week }\end{array}$} \\
\hline & & $\mathbf{M}$ & $\mathbf{F}$ & $\mathbf{M}$ & $\mathbf{F}$ & $\mathbf{M}$ & $\mathbf{F}$ & $\mathbf{M}$ & F & $\mathbf{M}$ & $\mathbf{F}$ \\
\hline 0-2 weeks & $\begin{array}{c}85 \\
595\end{array}$ & 3.5 & 3.3 & 1.50 & 1.60 & 359 & 381 & 0.34 & 0.36 & 0.34 & 0.36 \\
\hline 2-4 weeks & $\begin{array}{c}95 \\
665\end{array}$ & 4.1 & 3.8 & 1.44 & 1.55 & 343 & 370 & 0.32 & 0.35 & 0.32 & 0.35 \\
\hline 2 months & $\begin{array}{l}105 \\
735\end{array}$ & 5.4 & 5.0 & 1.20 & 1.30 & 288 & 311 & 0.27 & 0.29 & 0.27 & 0.29 \\
\hline 4 months & $\begin{array}{l}130 \\
910\end{array}$ & 7.0 & 6.4 & 1.15 & 1.26 & 275 & 301 & 0.26 & 0.28 & 0.26 & 0.28 \\
\hline 6 months & $\begin{array}{l}122 \\
854\end{array}$ & 7.9 & 7.3 & 0.96 & 1.04 & 229 & 247 & 0.22 & 0.23 & 0.22 & 0.23 \\
\hline
\end{tabular}

$\mathrm{Zn}$ shows the highest daily intake ( $359 \mu \mathrm{g} \mathrm{kg}^{-1} \mathrm{bw} /$ day for males and 381 for females at $0-2$ weeks), while $\mathrm{Cd}$ and $\mathrm{Pb}$ intake at 6 months was $0.22 \mu \mathrm{g} \mathrm{kg}{ }^{-1} \mathrm{bw} /$ week for males and 0.23 for females.

Table 8 reports daily $(\mathrm{Ni}$ and $\mathrm{Zn})$ and weekly $(\mathrm{Cd}$ and $\mathrm{Pb})$ safety limits and the related percentage health risk indexes.

Table 8. Percentage health risk index estimated for infants from 0 to 6 months for $\mathrm{Ni}, \mathrm{Zn}, \mathrm{Cd}$ and $\mathrm{Pb}$, reported separately for males (M) and females (F).

\begin{tabular}{|c|c|c|c|c|c|c|c|c|}
\hline \multirow{3}{*}{$\begin{array}{c}\text { Safety Limit } \\
\text { Age }\end{array}$} & \multicolumn{2}{|c|}{$\begin{array}{c}\mathrm{Ni} \\
2.8 \mu \mathrm{g} \mathrm{kg} \mathrm{kg}^{-1} \\
\text { bw/Day }\end{array}$} & \multicolumn{2}{|c|}{$\begin{array}{c}\mathrm{Zn} \\
7 \mathrm{mg} \mathrm{kg}^{-1} \\
\text { bw/Day }\end{array}$} & \multicolumn{2}{|c|}{$\begin{array}{c}\text { Cd } \\
2.5 \mu \mathrm{g} \mathrm{kg}^{-1} \\
\text { bw/week }\end{array}$} & \multicolumn{2}{|c|}{$\begin{array}{c}\mathrm{Pb} \\
3.5 \mu \mathrm{g} \mathrm{kg}^{-1} \\
\text { bw/Week }\end{array}$} \\
\hline & \multicolumn{8}{|c|}{ \% Health Risk Index } \\
\hline & $\mathbf{M}$ & F & $\mathbf{M}$ & F & $\mathbf{M}$ & F & $\mathbf{M}$ & F \\
\hline 0-2 weeks & 53.6 & 57.1 & 5.13 & 5.44 & 13.6 & 14.4 & 9.71 & 10.2 \\
\hline 2-4 weeks & 51.4 & 55.4 & 4.89 & 5.28 & 12.8 & 14.0 & 9.14 & 10.0 \\
\hline 2 months & 42.9 & 46.4 & 4.11 & 4.44 & 10.8 & 11.6 & 7.71 & 8.28 \\
\hline 4 months & 41.1 & 45.0 & 3.96 & 4.29 & 10.4 & 11.2 & 7.40 & 8.00 \\
\hline 6 months & 34.3 & 37.1 & 3.26 & 3.53 & 8.8 & 9.2 & 6.28 & 6.57 \\
\hline
\end{tabular}

The highest health risk indexes were obtained for $\mathrm{Ni}$, while the indexes for $\mathrm{Cd}$ always resulted lower than $15 \%$, those relating to $\mathrm{Pb}$ always lower than $10 \%$ and those for $\mathrm{Zn}$ about $5 \%$.

Figure 2 shows the mean concentrations of $\mathrm{Mn}$ in each of the 11 studied formulas together with the minima and maxima levels prescribed by EU and French regulations. 


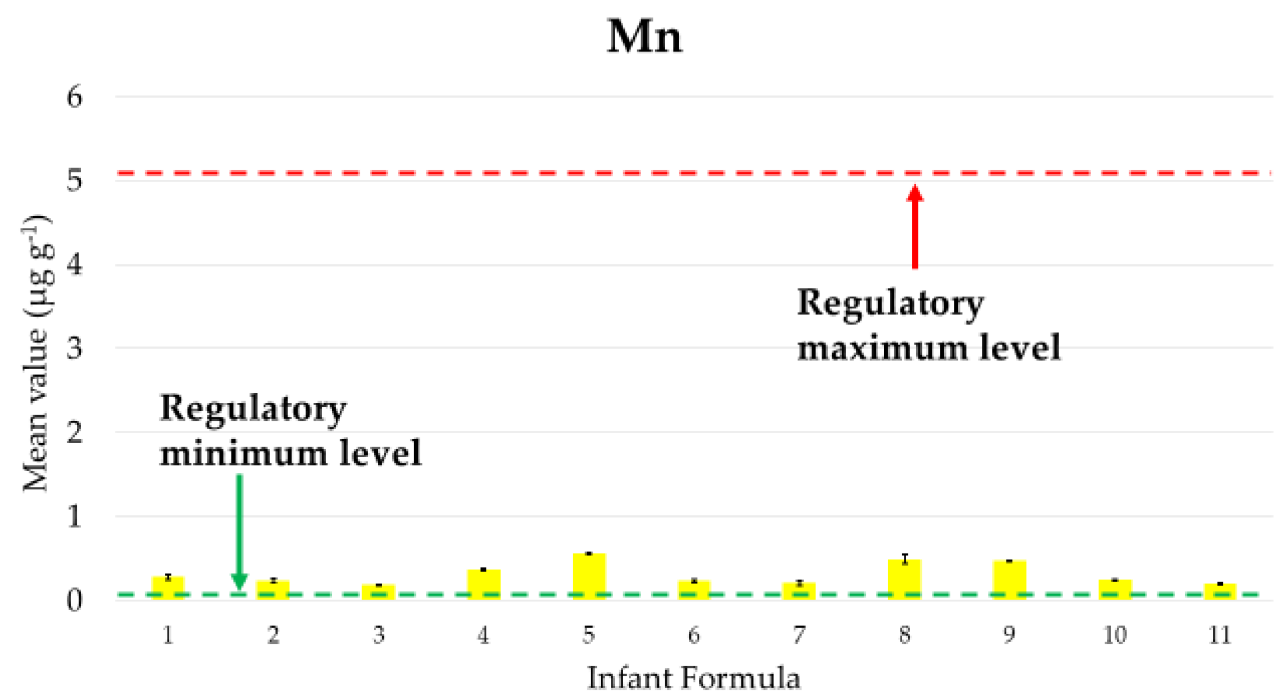

Figure 2. Mean values of the concentration, expressed in $\mu \mathrm{g} \mathrm{g}^{-1}$ for each formula, of Mn. The black line on each bar represents the standard deviation; the green and red lines represent, respectively, the regulatory minimum and maximum levels.

The Mn mean concentrations found in the studied formulas were always higher than the requested minimum level and much lower than the fixed maximum level in any product.

\section{Discussion}

The present study was conducted on 11 powdered formulas commercialized in Italy. A specific risk evaluation was performed for some toxic metals $(\mathrm{Cd}, \mathrm{Mn}, \mathrm{Ni}, \mathrm{Pb}$, and $\mathrm{Zn}$; $\mathrm{Cr}$, initially considered for the risk assessment, was lower than the LOD in more than 30\% of samples and, therefore, was excluded from this specific evaluation).

The first relevant result was related to the concentrations of the studied elements in the 11 formulas: 11 out of the 40 analytes (Be, B, Al, Zr, Nb, Sb, Te, W, V, Cr, and As) were lower than the LOD in more than $30 \%$ of cases. Among these elements, the levels of $\mathrm{Al}$ were the most surprising result because it was found to be lower than the LOD in $73 \%$ of the determinations. This finding is very positive from a food safety point of view because a chronic intake of $\mathrm{Al}$ via ingestion may negatively impact human health in the early stages of life [40]. Our results widely differ from those recovered previously, reporting Al levels as always higher than the LOD [41,42]. The origin of Al contamination in infant formula is not completely clear, but it probably derives from ingredients, packaging, and processing. Our result provides an indication of the improvement in the quality of food raw materials and of the production processes and food contact materials. Regarding other elements, the analytes' concentrations reported on the infant formula labels were very similar to those recovered by the present study, confirming its accuracy and usefulness for powdered infant formulas. Besides, in our knowledge, this is the first time that 40 elements were determined in powdered infant formulas. Thus, our results are original and could be used as benchmark data for future research because infant formulas play an essential role in those specific situations when breastfeeding is not possible. Indeed, about $16 \%$ of infants are not breastfed, while up to $75 \%$ are fed with both breast milk and formulas [43].

Another important finding is related to $\mathrm{Cd}, \mathrm{Mn} \mathrm{Ni}, \mathrm{Pb}$, and $\mathrm{Zn}$, which have been deeply studied due to their toxicity. In particular, $\mathrm{Zn}, \mathrm{Cd}$, and $\mathrm{Pb}$ levels ranged from 10.2 to 20.2 $\mu \mathrm{g} \mathrm{g}^{-1}$, from 0.001 to $0.006 \mu \mathrm{g} \mathrm{g}^{-1}$, and from 0.0006 to $0.0026 \mu \mathrm{g} \mathrm{g}^{-1}$, respectively. These results were in line with those reported by another study conducted in the European Union (EU) [44], which recovered $\mathrm{Zn}, \mathrm{Cd}$, and $\mathrm{Pb}$ range concentrations, respectively, equal to 36.5$52.3 \mu \mathrm{g} \mathrm{g}^{-1}, 0.0033-0.0045 \mu \mathrm{g} \mathrm{g}^{-1}$ and $0.0082-0.0439 \mu \mathrm{g} \mathrm{g}^{-1}$. Similarly, Bargellini et al. [45] reported concentrations for $\mathrm{Cd}, \mathrm{Pb}$, and $\mathrm{Zn}$ found in infant formula samples in the same 
order of magnitude. In contrast, other studies performed in countries outside the EU recovered very different levels. For example, a study in the field conducted in 2018 [30] on some infant formulas sold in Ethiopia reported a range concentration of $\mathrm{Zn}$ and $\mathrm{Pb}$ equal to $27.9-71.5 \mu \mathrm{g} \mathrm{g}^{-1}$ and $<\mathrm{LOD}-0.103 \mu \mathrm{g} \mathrm{g}^{-1}$, respectively, while Cd concentrations were always $<$ LOD. These differences in elemental concentrations all over the countries were also evidenced by other researches in China and Pakistan [46,47]. Likewise, similarities between the elemental concentrations found in formulas marketed in EU countries and differences with the levels reported for the formulas sold in countries outside EU are due to differences in raw materials, processing and packaging, and regulations. It should be essential to harmonize procedures and regulations worldwide to guarantee the same right to health, one of the internationally agreed human rights recognized by WHO.

As regards to $\mathrm{Mn}$, we found mean concentrations always lower than the maximum level prescribed by EU and French regulations for infant formulas. This result is in line with those reported for the levels of Mn recovered in 17 powdered infant or follow-on formulas purchased in France and 14 infant formula products purchased in the United States. In contrast, a soy-based infant formula and an amino acid-based medical infant formula exceeded $100 \mu \mathrm{g}$ of $\mathrm{Mn} / 100 \mathrm{kcal}[19,20]$.

In the present study, we also estimated the intake of $\mathrm{Cd}, \mathrm{Ni}, \mathrm{Pb}$, and $\mathrm{Zn}$ and assessed the related risk, according to gender and different periods from birth to 6 months of life; for this purpose, the estimated daily/weekly intake of $\mathrm{Cd}, \mathrm{Ni}, \mathrm{Pb}$ and $\mathrm{Zn}$ and their percentage health risk indexes were calculated. The results showed that the heavy metals intake from infant formulas was not so relevant and always lower than the safety limit. However, some heavy metals' ingestion occurs via powdered infant formula and this is not negligible, especially considering that the study population is highly susceptible. Besides, contaminants such as $\mathrm{Zn}, \mathrm{Cd}, \mathrm{Ni}$, and $\mathrm{Pb}$ are ubiquitous in the environment and can also be assumed through inhalation and skin absorption. Thus, the amount taken through infant formula represents just a part of the total intake, and it should be added to the quantities introduced into the body with air and with dermal contact. In addition, the contribution of the minerals and potentially toxic elements present in the water used for the reconstitution of the powdered formulas should be considered.

Similar risk evaluations have also been performed for breast milk, evidencing detectable levels of potential toxic elements such as $\mathrm{Pb}, \mathrm{Cd}, \mathrm{Cu}, \mathrm{As}, \mathrm{Zn}[27,48,49]$. All authors, however, agree on the great variability of the concentration of these elements due to mothers' dietary habits, lifestyles, occupational exposure, urban pollution exposure, lactation stage, etc. These interfering factors do not influence the elemental levels in infant formula products.

This study presents some limitations. First of all, the number of infant formulas was limited to 11, but these were the formulas authorized and commercialized in Italy when we collected the samples. Besides, we determine a very high number of elements for each studied formula with a unique analysis. Secondly, we estimated the dose of some toxic elements assumed by newborns through infant formula using the mean concentrations based on the analysis of only one lot for each formula. However, the manufacturing process of such products is strictly regulated by the specific international regulations (The Standard for Infant Formula and Formulas for Special Medical Purposes Intended for Infants CXS 72-1981); thus, differences among lots are necessarily below the acceptable quality limit. Finally, we assumed a theoretical weight; however, we performed the evaluation by considering different periods between the birth to the sixth month of life, and it was performed separately for males and females.

\section{Conclusions}

A determination of 40 elements in powdered infant formulas was performed. The concentration of the elements in 11 infant formulas authorized and commercialized in Italy demonstrated levels for some elements similar to those reported by other EU studies but different from those performed outside the EU. It is essential to eliminate these differences 
to guarantee the right to health for all the newborns all over the word. The specific risk assessment performed for $\mathrm{Cd}, \mathrm{Mn}, \mathrm{Ni}, \mathrm{Pb}$, and $\mathrm{Zn}$ demonstrated that the concentrations of these elements in the studied infant formulas were always below the considered limits; however, this issue must be constantly considered because the amount of potentially toxic elements assumed with infant formulas is just a part of all the quantity assumed (adding to the amount assumed via inhalation and dermal contact).

Author Contributions: Conceptualization, C.P. and M.V.; methodology, M.L.A. and A.A.; software, D.M. and V.C.; validation, M.L.A. and P.A.; formal analysis, E.M. and A.A.; investigation, D.M., V.C. and E.M.; resources, C.P. and S.C.; data curation, C.P.; writing-original draft preparation, M.L.A., D.M., V.C., E.M. and C.P.; writing-review and editing, A.A., C.P., M.L.A., P.A., S.C. and M.V.; project administration, C.P.; funding acquisition, C.P. and M.L.A. All authors have read and agreed to the published version of the manuscript.

Funding: This research was funded by SAPIENZA UNIVERSITY OF ROME, grant number RP11715C 819E4A20.

Institutional Review Board Statement: Not applicable because this study did not involve animals or humans.

Informed Consent Statement: Not applicable because this study did not involve humans.

Data Availability Statement: The data presented in this study are available on request from the corresponding author.

Conflicts of Interest: The authors declare no conflict of interest.

\section{References}

1. Regulation (EC) 178/2002 - Laying down the General Principles and Requirements of Food Law, Establishing the European Food Safety Authority and Laying down Procedures in Matters of Food Safety. Available online: https://eur-lex.europa.eu/eli/reg/20 02/178/oj (accessed on 13 October 2020).

2. World Health Organization-Food Safety. 2020. Available online: https://www.who.int/news-room/fact-sheets/detail/foodsafety (accessed on 13 October 2020).

3. Machado Nardi, V.A.; Auler, D.P.; Teixeira, R. Food safety in global supply chains: A literature review. J. Food Sci. 2020, 85, 883-891. [CrossRef] [PubMed]

4. Nobile, M.; Arioli, F.; Pavlovic, R.; Ceriani, F.; Lin, S.K.; Panseri, S.; Villa, R.; Chiesa, L.M. Presence of emerging contaminants in baby food. Food Addit. Contam. Part A 2020, 37, 131-142. [CrossRef] [PubMed]

5. Rollins, N.C.; Bhandari, N.; Hajeebhoy, N.; Horton, S.; Lutter, C.K.; Martines, J.C.; Piwoz, E.G.; Richter, L.M.; Victora, C.G. Why invest, and what it will take to improve breastfeeding practices? Lancet 2016, 387, 491-504. [CrossRef]

6. Arts, M.; Taqi, I.; Bégin, F. Improving the Early Initiation of Breastfeeding: The WHO-UNICEF Breastfeeding Advocacy Initiative. Breastfeed. Med. 2017, 12, 326-327. [CrossRef]

7. UNICEF. Breastfeeding: A Mother's Gift, for Every Child; UNICEF: New York, NY, USA, 2018.

8. World Health Organization-Infant and Young Child Feeding. 2020. Available online: https://www.who.int/news-room/factsheets/detail/infant-and-young-child-feeding (accessed on 13 April 2021).

9. Victora, C.G.; Bahl, R.; Barros, A.J.D.; França, G.V.A.; Horton, S.; Krasevec, J.; Murch, S.; Sankar, M.J.; Walker, N.; Rollins, N.C. Breastfeeding in the 21st century: Epidemiology, mechanisms, and lifelong effect. Lancet 2016, 387, 475-490. [CrossRef]

10. Regulation (EU) 609/2013 - On Food Intended for Infants and Young Children, Food for Special Medical Purposes, and Total Diet Replacement for Weight Control and Repealing Council Directive 92/52/EEC, Commission Directives 96/8/EC, 1999/21/EC, 2006/125/EC and 2006/141/EC, Directive 2009/39/EC of the European Parliament and of the Council and Commission Regulations (EC) No 41/2009 and (EC) No 953/2009. Available online: https:/ / eur-lex.europa.eu/eli/reg/2013/609/2017-07-11 (accessed on 13 April 2021).

11. Losio, M.N.; Pavoni, E.; Finazzi, G.; Agostoni, C.; Daminelli, P.; Dalzini, E.; Varisco, G.; Cinotti, S. Preparation of Powdered Infant Formula: Could Product's Safety Be Improved? J. Pediatr. Gastroenterol. Nutr. 2018, 67, 543-546. [CrossRef]

12. CODEX ALIMENTARIUS STANDARDS 72-1981—Standards for Infant Formula and Formulas for Special Medical Purposes Intended for Infants. Formerly CAC/RS 72-1972. Adopted as a Worldwide Standard in 1981. Amendment 1983, 1985, 1987, 2011, 2015 and 2016. Revision. 2007. Available online: http:/ /www.fao.org/fao-who-codexalimentarius/sh-proxy/en/?lnk=1\& url=https \%253A \%252F\%252Fworkspace.fao.org\%252Fsites\%252Fcodex\%252FStandards\%252FCXS\%2B72-1981\%252FCXS_07 2e.pdf (accessed on 13 April 2021).

13. Konings, E.J.; Roux, A.; Reungoat, A.; Nicod, N.; Campos-Giménez, E.; Ameye, L.; Bucheli, P.; Alloncle, S.; Dey, J.; Daix, G.; et al. Challenge to evaluate regulatory compliance for nutrients in infant formulas with current state-of-the-art analytical reference methods. Food Control 2020, 119, 107423. [CrossRef] 
14. Koletzko, B.; Baker, S.; Cleghorn, G.; Neto, U.F.; Gopalan, S.; Hernell, O.; Hock, Q.S.; Jirapinyo, P.; Lonnerdal, B.; Pencharz, P.; et al. Global standard for the composition of infant formula: Recommendations of an ESPGHAN coordinated international expert group. J. Pediatr. Gastroenterol. Nutr. 2005, 41, 584-599. [CrossRef]

15. Needleman, H.L.; Schell, A.; Bellinger, D.; Leviton, A.; Allred, E.N. The long-term effects of exposure to low doses of lead in childhood: An 11-year follow-up report. N. Engl. J. Med. 1990, 322, 83-88. [CrossRef]

16. Nevin, R. How lead exposure relates to temporal changes in IQ, crime and owed pregnancy. Environ. Res. 2000, 83, 1-22. [CrossRef]

17. Davidowski, L.; Grosser, Z.; Sarojam, P. Perkin Elmer Application Note. The Analysis of Baby Foods and Juices for Metals to Protect a Sensitive Population; Food Safety: Shelton, CT, USA, 2009. Available online: https://webcache.googleusercontent.com/search?q=cache: HIYVcJaWOsoJ:https:/ / www.medic-labor.sk/articles_blog/APP_AnalysisofBabyFoodsJuicesforMetal.pdf $+\& c d=1 \&$ hl=it\&ct= clnk\&gl=it\&client=firefox-b-d (accessed on 13 April 2021).

18. Hayano, M.; Nogawa, K.; Kido, T.; Kobayashi, E.; Honda, R.; Turitani, I. Dose-response relationship between urinary cadmium concentration and $\beta 2-$ microglobulinuria using logistic regression analysis. Arch. Environ. Occup. Health 1996, 51, 162-167. [CrossRef] [PubMed]

19. Mitchell, E.J.; Frisbie, S.H.; Roudeau, S.; Carmona, A.; Ortega, R. How much manganese is safe for infants? A review of the scientific basis of intake guidelines and regulations relevant to the manganese content of infant formulas. J. Trace Elem. Med. Biol. 2021, 65, 126710. [CrossRef]

20. Frisbie, S.H.; Mitchell, E.J.; Roudeau, S.; Domart, F.; Carmona, A.; Ortega, R. Manganese levels in infant formula and young child nutritional beverages in the United States and France: Comparison to breast milk and regulations. PLoS ONE 2019, 14, e0223636. [CrossRef] [PubMed]

21. Das, K.K.; Das, S.N.; Dhundasi, S.A. Nickel, its adverse health effects \& oxidative stress. Indian J. Med. Res. 2008, $128,412-425$.

22. Li, Y.; Xu, X.; Liu, J.; Wu, K.; Gu, C.; Shao, G.; Chen, S.; Chen, G.; Huo, X. The hazard of chromium exposure to neonates in Guiyu of China. Sci. Total Environ. 2008, 403, 99-104. [CrossRef] [PubMed]

23. Redgrove, J.; Rodriguez, I.; Mahadevan-Bava, S.; Exley, C. Prescription Infant Formulas Are Contaminated with Aluminium. Int. J. Environ. Res. Public Health 2019, 16, 899. [CrossRef] [PubMed]

24. ter Meulen, R.H. The ethical basis of the precautionary principle in health care decision making. Toxicol. Appl. Pharmacol. 2005, 207, 663-667. [CrossRef] [PubMed]

25. Decreto Ministeriale 17 Maggio 2016-Assistenza Sanitaria Integrativa per i Prodotti Inclusi nel Campo di Applicazione del Regolamento (UE) 609/2013 e per i Prodotti Alimentari Destinati ai Celiaci e Modifiche al Decreto 8 Giugno 2001. Available online: https:/ / www.gazzettaufficiale.it/eli/id/2016/06/13/16A04367/sg (accessed on 13 April 2021).

26. Astolfi, M.L.; Marconi, E.; Protano, C.; Vitali, M.; Schiavi, E.; Mastromarino, P.; Canepari, S. Optimization and validation of a fast digestion method for the determination of major and trace elements in breast milk by ICP-MS. Anal. Chim. Acta 2018, 1040, 49-62. [CrossRef] [PubMed]

27. Astolfi, M.L.; Protano, C.; Schiavi, E.; Marconi, E.; Capobianco, D.; Massimi, L.; Ristorini, M.; Baldassarre, M.E.; Laforgia, N.; Vitali, M.; et al. A prophylactic multi-strain probiotic treatment to reduce the absorption of toxic elements: In-vitro study and biomonitoring of breast milk and infant stools. Environ. Int. 2019, 130, 104818. [CrossRef] [PubMed]

28. Astolfi, M.L.; Marconi, E.; Protano, C.; Canepari, S. Comparative elemental analysis of dairy milk and plant-based milk alternatives. Food Control 2020, 116, 107327. [CrossRef]

29. Astolfi, M.L.; Protano, C.; Marconi, E.; Massimi, L.; Brunori, M.; Piamonti, D.; Migliara, G.; Vitali, M.; Canepari, S. A new treatment of human hair for elemental determination by inductively coupled mass spectrometry. Anal. Methods 2020b, 12, 1906-1918. [CrossRef]

30. Eticha, T.; Afrasa, M.; Kahsay, G.; Gebretsadik, H. Infant Exposure to Metals through Consumption of Formula Feeding in Mekelle, Ethiopia. Int. J. Anal. Chem. 2018, 2018, 2985698. [CrossRef]

31. World Health Organization. Child Growth Standards. Weight-for-Age. Available online: https://www.who.int/tools/childgrowth-standards/standards/weight-for-age (accessed on 13 April 2021).

32. European Food Safety Authority (EFSA). Scientific Report of EFSA. Cadmium dietary exposure in the European population. EFSA J. 2012, 10, 2551.

33. European Food Safety Authority (EFSA). CONTAM Panel. Statement on tolerable weekly intake for cadmium. EFSA J. 2011, 9, 1975.

34. European Food Safety Authority (EFSA). CONTAM Panel. Scientific opinion on lead in food. EFSA J. 2010, 8, 1570.

35. European Food Safety Authority (EFSA). Panel. Scientific Opinion on Dietary Reference Values for zinc. EFSA J. $2014,12,3844$.

36. European Food Safety Authority (EFSA). CONTAM Panel. Scientific opinion on the risks to public health related to the presence of chromium in food and drinking water. EFSA J. 2014, 12, 3595.

37. European Food Safety Authority (EFSA). CONTAM Panel. Scientific opinion on the risks to public health related to the presence of nickel in food and drinking water. EFSA J. 2015, 13, 4002. 
38. Arrêté du 11 Avril 2008 Relatif aux Préparations pour Nourrissons et aux Préparations de suite et Modifiant L'arrêté du 20 Septembre 2000 Relatif aux Aliments Diététiques Destinés à des fins Médicales Spéciales (Order of 11 April 2008 on Infant Formulas and Follow-On Formulas and Amending the Order of 20 September 2000 on Dietary Foods for Special Medical Purposes). n`0096 du 23 Avril 2008 Page 6700, Texte n¹8, Version Consolidée au 15 Janvier 2018 (n0096 of 23 April 2008 Page 6700, Text $n^{\circ} 18$, Consolidated Version on 15 January 2018). Journal Officiel de la République Française (Official Journal of the French Republic). Available online: https://www.legifrance.gouv.fr/affichTexte.do?cidTexte=JORFTEXT000018685743\& dateTexte $=20180115$ (accessed on 5 May 2021).

39. European Commission (EC). Commission Delegated Regulation (EU) 2016/127 of 25 September 2015 Supplementing Regulation (EU) No 609/2013 of the European Parliament and of the Council as Regards the Specific Compositional and Information Requirements for Infant Formula and Follow-on Formula and as Regards Requirements on Information Relating to Infant and Young Child Feeding. Official Journal of the European Union. 2015. Available online: https://eur-lex.europa.eu/legal-content/ EN/TXT/?uri=CELEX\%3A32016R0127 (accessed on 5 May 2021).

40. Corkins, M.R. Committee on Nutrition. Aluminum Effects in Infants and Children. Pediatrics 2019, 144, e20193148. [CrossRef] [PubMed]

41. Igweze, Z.N.; Ekhator, O.C.; Nwaogazie, I.; Orisakwe, O.E. Public Health and Paediatric Risk Assessment of Aluminium, Arsenic and Mercury in Infant Formulas Marketed in Nigeria. Sultan Qaboos Univ. Med. J. 2020, 20, e63-e70. [CrossRef] [PubMed]

42. Chuchu, N.; Patel, B.; Sebastian, B.; Exley, C. The aluminium content of infant formulas remains too high. BMC Pediatr. 2013, 13, 162. [CrossRef] [PubMed]

43. Centers for Disease Control and Prevention. Facts. Key Breastfeeding Indicators. Available online: https://www.cdc.gov/ breastfeeding/data/facts.html (accessed on 5 May 2021).

44. Pandelova, M.; Lopez, W.L.; Michalke, B.; Schramm, K.-W. Ca, Cd, Cu, Fe, Hg, Mn, Ni, Pb, Se, and Zn contents in baby foods from the EU market: Comparison of assessed infant intakes with the present safety limits for minerals and trace elements. J. Food Compost. Anal. 2012, 27, 120-127. [CrossRef]

45. Bargellini, A.; Venturelli, F.; Casali, E.; Ferrari, A.; Marchesi, I.; Borella, P. Trace elements in starter infant formula: Dietary intake and safety assessment. Environ. Sci. Pollut. Res. Int. 2018, 25, 2035-2044. [CrossRef]

46. Su, C.; Zheng, N.; Gao, Y.; Huang, S.; Yang, X.; Wang, Z.; Yang, H.; Wang, J. Content and Dietary Exposure Assessment of Toxic Elements in Infant Formulas from the Chinese Market. Foods 2020, 9, 1839. [CrossRef] [PubMed]

47. Akhtar, S.; Shahzad, M.A.; Yoo, S.H.; Ismail, A.; Hameed, A.; Ismail, T.; Riaz, M. Determination of Aflatoxin $\mathrm{M}_{1}$ and Heavy Metals in Infant Formula Milk Brands Available in Pakistani Markets. Korean J. Food Sci. Anim. Resour. 2017, 37, 79-86. [CrossRef] [PubMed]

48. Vollset, M.; Iszatt, N.; Enger, Ø.; Gjengedal, E.L.F.; Eggesbø, M. Concentration of mercury, cadmium, and lead in breast milk from Norwegian mothers: Association with dietary habits, amalgam and other factors. Sci. Total Environ. 2019, 677, 466-473. [CrossRef] [PubMed]

49. Aumeistere, L.; Ciproviča, I.; Zavadska, D.; Bavrins, K.; Borisova, A. Zinc Content in Breast Milk and Its Association with Maternal Diet. Nutrients 2018, 10, 1438. [CrossRef] [PubMed] 